\title{
BERGMAN PROJECTION INDUCED BY KERNEL WITH INTEGRAL REPRESENTATION
}

\author{
JOSÉ ÁNGEL PELÁEZ, JOUNI RÄTTYÄ, AND BRETT D. WICK
}

\begin{abstract}
Bounded Bergman projections $P_{\omega}: L_{\omega}^{p}(v) \rightarrow L_{\omega}^{p}(v)$, induced by reproducing kernels admitting the representation

$$
\frac{1}{(1-\bar{z} \zeta)^{\gamma}} \int_{0}^{1} \frac{d \nu(r)}{1-r \bar{z} \zeta}
$$

and the corresponding (1,1)-inequality are characterized in terms of Bekollé-Bonami-type conditions. The two-weight inequality for the maximal Bergman projection $P_{\omega}^{+}: L_{\omega}^{p}(u) \rightarrow$ $L_{\omega}^{p}(v)$ in terms of Sawyer-testing conditions is also discussed.
\end{abstract}

\section{INTRODUCTION AND MAIN RESULTS}

Let $\widehat{\mathcal{D}}$ denote the set of positive Borel measures $\omega$ on $[0,1)$ such that $\widehat{\omega}(r)=\int_{r}^{1} d \omega(r) \leq$ $C \widehat{\omega}\left(\frac{1+r}{2}\right)$ for some $C=C(\omega)>0$. For $0<p<\infty$ and $\omega \in \widehat{\mathcal{D}}$, the weighted Bergman space $A_{\omega}^{p}$ consists of analytic functions $f$ in the unit disc $\mathbb{D}=\{z \in \mathbb{C}:|z|<1\}$ such that

$$
\|f\|_{A_{\omega}^{p}}^{p}=\int_{\mathbb{D}}|f(z)|^{p} d(\omega \otimes m)(z)<\infty,
$$

where $d(\omega \otimes m)\left(r e^{i \theta}\right)=r d \omega(r) d \theta$. As usual, we write $A_{\alpha}^{p}$ for the standard weighted Bergman space induced by the measure $\omega$ for which $d(\omega \otimes m)(z)=(\alpha+1)\left(1-|z|^{2}\right)^{\alpha} d A(z)=d A_{\alpha}(z)$, where $-1<\alpha<\infty$ and $d A(z)=\frac{d x d y}{\pi}$ denotes the normalized Lebesgue area measure on $\mathbb{D}$. For simplicity, we also write $\int_{E} f(z) d(\omega \otimes m)(z)=(f \omega)(E)$ for each non-negative $f$.

By the proof of [14, Theorem 3.3], for $\omega \in \widehat{\mathcal{D}}$, the norm convergence in $A_{\omega}^{2}$ implies the uniform convergence on compact subsets, and hence each point evaluation $L_{z}(f)=f(z)$ is a bounded linear functional in the Hilbert space $A_{\omega}^{2}$. Therefore there exist unique reproducing kernels $B_{z}^{\omega} \in A_{\omega}^{2}$ with $\left\|L_{z}\right\|=\left\|B_{z}^{\omega}\right\|_{A_{\omega}^{2}}$ such that

$$
f(z)=\left\langle f, B_{z}^{\omega}\right\rangle_{A_{\omega}^{2}}=\int_{\mathbb{D}} f(\zeta) \overline{B_{z}^{\omega}(\zeta)} d(\omega \otimes m)(\zeta), \quad f \in A_{\omega}^{2} .
$$

The Bergman projection

$$
P_{\omega}(f)(z)=\int_{\mathbb{D}} f(\zeta) \overline{B_{z}^{\omega}(\zeta)} d(\omega \otimes m)(\zeta)
$$

is an orthogonal projection from $L_{\omega}^{2}$ to $A_{\omega}^{2}$ and it is closely related to the maximal Bergman projection

$$
P_{\omega}^{+}(f)(z)=\int_{\mathbb{D}} f(\zeta)\left|B_{z}^{\omega}(\zeta)\right| d(\omega \otimes m)(\zeta)
$$

For a positive Borel measure $\omega$ on $[0,1)$, a positive $(\omega \otimes m)$-integrable function $v$ is called an $\omega$-weight. If $\omega \otimes m$ is the normalized Lebesgue area measure, then an $\omega$-weight is simply

Date: July 2, 2018.

Key words and phrases. Bergman space, Bergman Projection, doubling weight, dyadic operator, reproducing kernel, testing condition.

This research was supported in part by the Ramón y Cajal program of MICINN (Spain); by Ministerio de Economía y Competitivivad, Spain, project MTM2014-52865-P; by La Junta de Andalucía, (FQM210) and (P09-FQM-4468); by Academy of Finland project no. 268009, and by Faculty of Science and Forestry of University of Eastern Finland project no. 930349; by National Science Foundation Grants DMS \# 1560955 and \# 1603246. 
called a weight. For $0<p<\infty$ and an $\omega$-weight $v$, the Lebesgue space $L_{\omega}^{p}(v)$ consists of $f$ such that

$$
\|f\|_{L_{\omega}^{p}(v)}^{p}=\int_{\mathbb{D}}|f(z)|^{p} v(z) d(\omega \otimes m)(z)<\infty .
$$

The boundedness of projections on $L^{p}$-spaces is an intriguing topic which presents obvious mathematical difficulties and has plenty of applications in operator theory [1, 3, 4, 6, 7, 12, 15, 23, 24]. It is known that for $1<p<\infty$ and $d(\omega \otimes m)=d A_{\alpha}$,

$$
\left\|P_{\omega}(f)\right\|_{L_{\omega}^{p}(v)} \leq C\|f\|_{L_{\omega}^{p}(v)}, \quad f \in L_{\omega}^{p}(v),
$$

if and only if $v$ satisfies the Bekollé-Bonami condition

$$
B_{p, \alpha}(v)=\sup _{S} \frac{v A_{\alpha}(S)\left(v^{\frac{-p^{\prime}}{p}} A_{\alpha}(S)\right)^{\frac{p}{p^{\prime}}}}{\left.\left(A_{\alpha}(S)\right)\right)^{p}}<\infty,
$$

where the supremum is taken over all the Carleson squares $S$ in $\mathbb{D}$ 3, 4. In the above result $P_{\alpha}$ can be replaced by the maximal Bergman projection $P_{\alpha}^{+}\left[3,4\right.$, and $\left\|P_{\alpha}^{+}\right\| \lesssim B_{p, \alpha}(v)^{\max \left\{1, \frac{1}{p-1}\right\}}$ by [15. It is also known [4, 5, 7] that the weak $(1,1)$ inequality

is equivalent to

$$
v A_{\alpha}\left(\left\{z \in \mathbb{D}:\left|P_{\alpha}(f)(z)\right|>\lambda\right\}\right) \leq C \frac{\|f\|_{L_{\alpha}^{1}(v)}}{\lambda}
$$

for the weighted maximal function

$$
M_{\alpha}(v)(z) \leq C v(z), \quad \text { a.e. } z \in \mathbb{D},
$$

$$
M_{\alpha}(v)(z)=\sup _{z \in D(a, r)} \frac{v A_{\alpha}(D(a, r) \cap \mathbb{D})}{A_{\alpha}(D(a, r) \cap \mathbb{D})}, \quad z \in \mathbb{D} .
$$

Here $D(a, r)$ denotes the Euclidean disc of center $a$ and radius $r$.

An immediate difficulty in controlling (1.1) for a given measure $\omega \in \widehat{\mathcal{D}}$ is the lack of an explicit expression for the Bergman kernel $B_{z}^{\omega}$. Writing $\omega_{x}=\int_{0}^{1} r^{x} \omega(r) d r$, the normalized monomials $z^{n} / \sqrt{2 \omega_{2 n+1}}$ form the standard orthonormal basis of $A_{\omega}^{2}$, and hence

$$
B_{z}^{\omega}(\zeta)=\sum_{n=0}^{\infty} \frac{(\zeta \bar{z})^{n}}{2 \omega_{2 n+1}}, \quad z, \zeta \in \mathbb{D} .
$$

This formula and a decomposition norm theorem was recently used to obtain a precise estimate for the $L_{v}^{p}$-integral of $B_{z}^{\omega}$ when $v, \omega$ are weights in $\widehat{\mathcal{D}}[12$, Theorem 1]. With the aid of these estimates, (1.1) was characterized in the case when $\omega$ and $v$ are weights in the class $\mathcal{R}$ [12, 13. A positive Borel measure $\omega$ on $[0,1)$ belongs to $\mathcal{R}$, if there exist $C=C(\omega)>0, \gamma=\gamma(\omega)>0$ and $\beta=\beta(\omega) \geq \gamma$ such that

$$
C^{-1}\left(\frac{1-r}{1-t}\right)^{\gamma} \widehat{\omega}(t) \leq \widehat{\omega}(r) \leq C\left(\frac{1-r}{1-t}\right)^{\beta} \widehat{\omega}(t), \quad 0 \leq r \leq t<1 .
$$

In view of the above results two immediate questions arise. First, is it possible to extend the classical Bekollé-Bonami's results to projections $P_{\omega}$ induced by measures in $\widehat{\mathcal{D}}$ ? Second, is it possible to consider other weights than just those in $\mathcal{R}$ in the same spirit as in [12, Theorem 3]?

A natural approach to these question is to employ tools from harmonic analysis. However, it seems that to do so one needs the Bergman kernel $B_{z}^{\omega}$ to have some structure. The first result of this study shows that certain doubling measures induce kernels with suitable properties for our purposes.

Theorem 1. Let $\nu$ be a finite positive measure supported on $[0,1]$ such that $\int_{0}^{1} \frac{d \nu(r)}{1-r}$ diverges. Then there exists $\omega \in \widehat{\mathcal{D}}$ such that

$$
B_{z}^{\omega}(\zeta)=\frac{1}{1-\bar{z} \zeta} \int_{0}^{1} \frac{d \nu(r)}{1-r \bar{z} \zeta}, \quad z, \zeta \in \mathbb{D}
$$


Since each kernel $B_{z}^{\omega}$ induced by $\omega \in \widehat{\mathcal{D}}$ has the representation (1.3), and

$$
\frac{1}{1-z} \int_{0}^{1} \frac{d \nu(r)}{1-r z}=\sum_{n=0}^{\infty}\left(\int_{0}^{1} \frac{1-r^{n+1}}{1-r} d \nu(r)\right) z^{n},
$$

the proof of Theorem 1 basically boils down to solving a Hausdorff moment problem. In Section 2 we will prove a more general result from which Theorem 1 immediately follows.

Next we focus on extending the classical Bekolle-Bonami's results for those measures $\omega \in \mathcal{R}$ that induce kernels admitting the representation

$$
B_{z}^{\omega}(\zeta)=\frac{1}{(1-\bar{z} \zeta)^{\gamma}} \int_{0}^{1} \frac{d \nu(r)}{1-r \bar{z} \zeta}, \quad z, \zeta \in \mathbb{D}
$$

for some $\gamma \geq 1$. For $1<p<\infty$ and $\omega \in \widehat{\mathcal{D}}$, an $\omega$-weight $v$ belongs $B_{p, \omega}$ if

$$
B_{p, \omega}(v)=\sup _{S} \frac{(v \omega)(S)}{\omega(S)}\left(\frac{\left(v^{-\frac{p^{\prime}}{p}} \omega\right)(S)}{\omega(S)}\right)^{\frac{p}{p^{\prime}}}<\infty .
$$

Theorem 2. Let $1<p<\infty$ and $\omega \in \mathcal{R}$ such that $B_{z}^{\omega}$ admits the representation

$$
B_{z}^{\omega}(\zeta)=\frac{1}{(1-\bar{z} \zeta)^{\gamma}} \int_{0}^{1} \frac{d \nu(r)}{1-r \bar{z} \zeta}, \quad z, \zeta \in \mathbb{D}
$$

for some $\gamma \geq 1$ and a positive measure $\nu$ supported on $[0,1]$. For an $\omega$-weight $v$, the following statements are equivalent:

(i) $P_{\omega}^{+}: L_{\omega}^{p}(v) \rightarrow L_{\omega}^{p}(v)$ is bounded;

(ii) $P_{\omega}: L_{\omega}^{p}(v) \rightarrow L_{\omega}^{p}(v)$ is bounded;

(iii) $P_{\omega}: L_{\omega}^{p}(v) \rightarrow L_{\omega}^{p, \infty}(v)$ is bounded;

(iv) $v \in B_{p, \omega}$.

Moreover,

$$
\left\|P_{\omega}^{+}\right\|_{L_{\omega}^{p}(v) \rightarrow L_{\omega}^{p}(v)} \lesssim B_{p, \omega}(v)^{\max \left\{1, \frac{1}{p-1}\right\} .}
$$

To prove (iii) $\Rightarrow$ (iv) in Theorem 2 , we estimate $\left|B_{z_{0}}^{\omega}(\zeta)-B_{z}^{\omega}(\zeta)\right|$ upwards for suitable chosen $z, z_{0}, \zeta$, and we also establish the useful relation

$$
\int_{0}^{1} \frac{d \nu(r)}{1-r x} \asymp \frac{(1-x)^{\gamma-1}}{\widehat{\omega}(x)}, \quad x \in[0,1),
$$

for the measures $\nu$ and $\omega$. The proof of (iv) $\Rightarrow(\mathrm{i})$ is based on known ideas of controlling $P_{\omega}^{+}$by two discrete dyadic operators [8, 9, 15], and it is done in the case of a more general operator. Theorem 2 is proved in Section 3 ,

Now we turn to study of the weak $(1,1)$-inequality. For a positive Borel measure $\omega$ on $[0,1)$, the weighted maximal function of $f \in L_{\omega}^{1}$ is

$$
M_{\omega}(f)(z)=\sup _{z \in D(a, r)} \frac{\int_{D(a, r) \cap \mathbb{D}}|f(\zeta)| d(\omega \otimes m)(\zeta)}{\omega(D(a, r) \cap \mathbb{D})}, \quad z \in \mathbb{D} .
$$

A non-negative function $v \in L_{\omega, \text { loc }}^{1}$ belongs to $B_{1, \omega}$ if there exists a constant $C=C(v, \omega)>0$ such that

$$
M_{\omega}(v)(z)=\sup _{a: z \in D(a, r)} \frac{\int_{D(a, r) \cap \mathbb{D}} v(\zeta) d(\omega \otimes m)(\zeta)}{\omega(D(a, r) \cap \mathbb{D})} \leq C v(z)
$$

for almost every $z \in \mathbb{D}$. The infimum of such constants is denoted by $B_{1, \omega}(v)$. In order to obtain the weak $(1,1)$-inequality we use the classical Calderón-Zygmund decomposition for functions in $L_{\omega}^{1}$. This causes the extra hypothesis on $\omega$ appearing in the statement of the following result, the proof of which is given in Section 4 . 
Theorem 3. Let $\omega \in \mathcal{R}$ be such that $\omega([a, b]) \asymp \omega\left(\left[a, \frac{a+b}{2}\right]\right) \asymp \omega\left(\left[\frac{a+b}{2}, b\right]\right)$ for all $0 \leq a, b \leq 1$ and $B_{z}^{\omega}$ admits the representation (1.5) for some $\gamma \geq 1$ and a positive measure $\nu$ supported on $[0,1]$. For a $\omega$-weight $v$, the following statements are equivalent:

(i) $P_{\omega}^{+}: L_{\omega}^{1}(v) \rightarrow L_{\omega}^{1, \infty}(v)$ is bounded;

(ii) $P_{\omega}: L_{\omega}^{1}(v) \rightarrow L_{\omega}^{1, \infty}(v)$ is bounded;

(iii) $v \in B_{1, \omega}$.

In Theorems 2 and 3 one of the essential hypothesis is $\omega \in \mathcal{R}$ while Theorem 1 concerns measures in $\widehat{\mathcal{D}}$. However, if $\gamma$ appearing in (1.5) is strictly larger than one, then $\omega \in \mathcal{R}$ by Lemma 10 below. It is also worth noticing that kernels admitting the representation (1.5) with $\gamma=1$ and their connection to logarithmically subharmonic weights have been discussed earlier in [19], and the starting point for our consideration towards Theorem 1 has similarities with arguments used there.

The two-weight inequality $\left\|P^{+}(f)\right\|_{L_{u}^{p}} \leq C\|f\|_{L_{v}^{p}}$ was recently characterized in terms of testing conditions on the indicators of Carleson squares [2]. The last of our main results offers a generalization of this result to the class of radial weights with kernels of the form (1.5). We write $1_{E}$ for the characteristic function of the set $E$, and write $\mathcal{M}_{h}$ for the multiplication operator $\mathcal{M}_{h}(f)=f h$.

Theorem 4. Let $1<p<\infty$, and let $\omega$ be a finite positive measure on $[0,1]$ such that $B_{z}^{\omega}$ admits the representation (1.5) for some $\gamma \geq 1$ and a positive measure $\nu$ supported on $[0,1]$. Let $v, u$ be $\omega$-weights and denote $\sigma=v^{1-p^{\prime}}$. Then $P_{\omega}^{+}: L_{\omega}^{p}(v) \rightarrow L_{\omega}^{p}(u)$ is bounded if and only if there exist constants $C_{0}=C_{0}(p, v, u, \omega)>0$ and $C_{0}^{\star}=C_{0}^{\star}(p, v, u, \omega)>0$ such that

$$
\left\|\mathcal{M}_{u^{1 / p}} P_{\omega}^{+} \mathcal{M}_{\sigma^{1 / p^{\prime}}}\left(1_{S} \sigma^{1 / p}\right)\right\|_{L_{\omega}^{p}} \leq C_{0}\left\|1_{S} \sigma^{1 / p}\right\|_{L_{\omega}^{p}}
$$

and

$$
\left\|\mathcal{M}_{\sigma^{1 / p^{\prime}}} P_{\omega}^{+} \mathcal{M}_{u^{1 / p}}\left(1_{S} u^{1 / p^{\prime}}\right)\right\|_{L_{\omega}^{p^{\prime}}} \leq C_{0}^{\star}\left\|1_{S} u^{1 / p^{\prime}}\right\|_{L_{\omega}^{p^{\prime}}}
$$

for all Carleson squares $S \subset \mathbb{D}$. Moreover, there exists a constant $C_{1}=C_{1}(p, \omega)>0$ such that

$$
\left\|P_{\omega}^{+}\right\|_{L_{\omega}^{p}(v) \rightarrow L_{\omega}^{p}(u)} \leq C_{1}\left(C_{0}+C_{0}^{\star}\right) .
$$

Theorem 4 is deduced from a more general result in Section 5 ,

\section{Integral FORMUla FOR THE BERGMAN KERNEL}

The solution of the Hausdorff moment problem says that for a given sequence $\left\{m_{n}\right\}_{n=0}^{\infty}$ of positive numbers there exists a positive Borel measure supported on $[0,1]$ such that

$$
m_{n}=\int_{0}^{1} s^{n} d \mu(s), \quad n \in \mathbb{N} \cup\{0\},
$$

if and only if, the sequence is completely monotonic i.e. $(-1)^{k}\left(\Delta^{k} m\right)_{n} \geq 0$, where $(\Delta m)_{n}=$ $m_{n+1}-m_{n}$ is the discrete difference operator and

$$
\left(\Delta^{k} m\right)_{n}=\left(\Delta \Delta^{k-1} m\right)_{n}=\left(\Delta^{k-1} m\right)_{n+1}-\left(\Delta^{k-1} \Delta m\right)_{n}, \quad k \in \mathbb{N} \backslash\{1\} .
$$

A function $f$ is completely monotonic on $[0, \infty)$, if

$$
(-1)^{k} f^{(k)}(x) \geq 0, \quad x>0, \quad k \in \mathbb{N} \cup\{0\},
$$

and $f:[0, \infty) \rightarrow[0, \infty)$ is Bernstein, if

$$
(-1)^{k} f^{(k)}(x) \leq 0, \quad x>0, \quad k \in \mathbb{N} .
$$

The first two of the following basic properties are easy to verify, for the third and fourth ones, see [18, Theorem 3.7] and [22, Theorem 1], respectively:

(1) If $f_{1}$ and $f_{2}$ are completely monotonic functions, so are $f_{1}+f_{2}$ and $f_{1} f_{2}$;

(2) If $f_{1}$ and $f_{2}$ are Bernstein functions, so is $f_{1}+f_{2}$; 
(3) If $f_{1}$ is completely monotonic and $f_{2}$ is a Bernstein function, then $f_{1} \circ f_{2}$ is a completely monotonic function;

(4) If $f$ is completely monotonic, then $\{f(a+n)\}_{n=0}^{\infty}$ is a completely monotonic sequence for each $a>0$.

Theorem 1 follows from the following result.

Theorem 5. Let $F:[0, \infty) \rightarrow(0, \infty)$ be a $C^{\infty}$-function and $\varphi(z)=\sum_{n=0}^{\infty} \widehat{\varphi}(n) z^{n}$ a non-trivial analytic function such that $1 / F$ is completely monotonic and $F(a+2 n+1)=\sum_{j=0}^{n} \widehat{\varphi}(j)$ for some $a \in(0, \infty)$ and all $n \in \mathbb{N} \cup\{0\}$. Then there exists a positive Borel measure $\omega$ on $[0,1]$ such that

$$
\frac{\varphi(z)}{1-z}=\sum_{k=0}^{\infty} \frac{z^{k}}{2 \omega_{2 k+1}}, \quad z \in \mathbb{D} .
$$

Moreover, if $\lim _{n \rightarrow \infty} F(a+2 n+1)=\infty, F(a+2 n) \lesssim F(a+n)$ and there exists a positive constant $M>1$ such that $\lim _{n \rightarrow \infty} \frac{M^{n}}{F(a+n)}=\infty$, then $\omega \in \widehat{\mathcal{D}}$ and

$$
\frac{\varphi(\bar{z} \zeta)}{1-\bar{z} \zeta}=B_{z}^{\omega}(\zeta), \quad \zeta, z \in \mathbb{D}
$$

Proof. Since $1 / F$ is completely monotonic, there exists a positive Borel measure $\omega$ on $[0,1]$ such that $F(a+m)=\frac{1}{2 \omega_{m}}$ for all $m \in \mathbb{N} \cup\{0\}$. In particular, $F(a+2 n-1)=\frac{1}{2 \omega_{2 n-1}}$ for all $n \in \mathbb{N}$. Therefore

$$
\begin{aligned}
\frac{\varphi(z)}{1-z} & =\frac{1}{z}\left(\sum_{k=1}^{\infty} z^{k}\right)\left(\sum_{j=0}^{\infty} \widehat{\varphi}(j) z^{j}\right)=\frac{1}{z} \sum_{n=1}^{\infty}\left(\sum_{j=0}^{n-1} \widehat{\varphi}(j)\right) z^{n} \\
& =\sum_{n=1}^{\infty} F(a+2 n-1) z^{n-1}=\sum_{n=1}^{\infty} \frac{z^{n-1}}{2 \omega_{2 n-1}}=\sum_{k=0}^{\infty} \frac{z^{k}}{2 \omega_{2 k+1}},
\end{aligned}
$$

and thus (2.2) is proved. Moreover,

$$
\omega(\{1\}) \leq \omega\left(\left[1-\frac{1}{2 n-1}, 1\right]\right) \lesssim \omega_{2 n-1}=\frac{1}{2 F(a+2 n-1)} \rightarrow 0, \quad n \rightarrow \infty,
$$

and if $m=\frac{1}{M-1}+1$, then there exists a constant $C=C(\omega)>0$ such that

$$
\widehat{\omega}(0) \leq C \widehat{\omega}\left(1-\frac{1}{m}\right) .
$$

For otherwise we would have $\widehat{\omega}\left(1-\frac{1}{m}\right)=0$, and then

$$
\frac{1}{2 F(a+n)}=\omega_{n}=\int_{0}^{1-\frac{1}{m}} r^{n} d \omega(r) \leq\left(1-\frac{1}{m}\right)^{n} \int_{0}^{1-\frac{1}{m}} d \omega(r)=M^{-n} \int_{0}^{1-\frac{1}{m}} d \omega(r),
$$

which yields a contradiction with the hypothesis $\lim _{n \rightarrow \infty} \frac{M^{n}}{F(a+n)}=\infty$. Since $\omega_{n}=\frac{1}{2 F(a+n)} \lesssim$ $\frac{1}{2 F(a+2 n)}=\omega_{2 n}$, this together with (2.3) and (2.4) implies $\omega \in \widehat{\mathcal{D}}$ by [14, Lemma 2.1].

Proof of Theorem 1. Consider the function $\varphi(z)=\int_{0}^{1} \frac{d \nu(r)}{1-r z}=\sum_{j=0}^{\infty} \nu_{j} z^{j}$, and observe that $\sum_{j=0}^{n} \nu_{j}=\int_{0}^{1} \frac{1-r^{n+1}}{1-r} d \nu(r)=F(2 n+1+1 / 2)$ for

$$
F(x)=\int_{0}^{1} \frac{1-r^{\frac{x+\frac{1}{2}}{2}}}{1-r} d \nu(r), \quad 0 \leq x<\infty .
$$

Since $f(x)=1 / x$ is completely monotonic and $F$ is a Bernstein function as is seen by direct calculations, $1 / F$ is completely monotonic. Therefore, by Theorem 5 , there exists a positive Borel measure $\omega$ on $[0,1]$ such that

$$
\frac{1}{1-z} \int_{0}^{1} \frac{d \nu(r)}{1-r z}=\sum_{k=0}^{\infty} \frac{z^{k}}{2 \omega_{2 k+1}}, \quad z \in \mathbb{D} .
$$


Moreover, $\omega$ is supported on $[0,1)$ because $\sum_{j=0}^{\infty} \nu_{j}=\infty$, and it satisfies (2.4) because $\lim _{n \rightarrow \infty} \frac{M^{n}}{\int_{0}^{1} \frac{1-r^{n+1}}{1-r} d \nu(r)} \geq \lim _{n \rightarrow \infty} \frac{M^{n}}{n \nu([0,1])}=\infty$. Since $1-r^{\frac{2 m+1}{2}} \leq 2\left(1-r^{\frac{m+1}{2}}\right)$ for all $m \in \mathbb{N} \cup\{0\}$, we also have $\omega_{m} \lesssim \omega_{2 m}$. Hence $\omega \in \widehat{\mathcal{D}}$ by [14, Lemma 2.1], and

$$
B_{z}^{\omega}(\zeta)=\frac{1}{1-\bar{z} \zeta} \int_{0}^{1} \frac{d \nu(r)}{1-r \bar{z} \zeta}, \quad z, \zeta \in \mathbb{D}
$$

Theorems 1 and 5 can be used to provide examples of concrete Bergman reproducing kernels:

(1) If $\nu$ is the Lebesgue measure, Theorem 1 gives the kernel

$$
B_{z}^{\omega}(\zeta)=\frac{1}{1-\bar{z} \zeta} \frac{1}{\bar{z} \zeta} \log \frac{1}{1-\bar{z} \zeta} .
$$

(2) Theorem 5 allows to recover the well-known formula of the Bergman kernels induced by the standard weights $\omega(z)=(\alpha+1)\left(1-|z|^{2}\right)^{\alpha}, \alpha>-1$. Indeed, by choosing $a=1$ and $F(x)=\frac{1}{\beta(x / 2, \alpha+1)}$, have $F(a+2 j+1)=F(2 j+2)=\frac{1}{\beta(j+1, \alpha+1)}$. It is clear that $1 / F$ is completely monotonic on $[0, \infty)$ and the function $\varphi$ associated to $F$ is

$$
\frac{1}{(1-z)^{\alpha+1}}=\frac{1}{\beta(1, \alpha+1)}+\sum_{j=1}^{\infty}\left(\frac{1}{\beta(j+1, \alpha+1)}-\frac{1}{\beta(j, \alpha+1)}\right) z^{j} .
$$

(3) Let $\varphi(z)=\frac{\log \frac{e}{1-z}}{1-z}=1+\sum_{j=1}^{\infty}\left(1+\sum_{k=1}^{j} \frac{1}{k}\right) z^{j}$ so that $\sum_{j=0}^{n} \widehat{\varphi}(j)=1+(n+$ 1) $\int_{0}^{1} \frac{1-s^{n+1}}{1-s} d s$, and choose $a=\frac{1}{2}$ and $F(x)=1+\frac{x+1}{2} \int_{0}^{1} \frac{1-s^{\frac{x+\frac{1}{2}}{2}}}{1-s} d s$ so that $F(1 / 2+$ $2 n+1)=\sum_{j=0}^{n} \widehat{\varphi}(j)$. Since $x \rightarrow \frac{x+1}{2}$ and $x \rightarrow \int_{0}^{1} \frac{1-s \frac{x+\frac{1}{2}}{2}}{1-s} d s$ are Bernstein functions on $[0, \infty), F$ is completely monotonic on $[0, \infty)$. Moreover, it is clear that $F$ satisfies the rest of hypothesis of Theorem $[5$, and hence there exists $\omega \in \widehat{\mathcal{D}}$ such that

$$
B_{z}^{\omega}(\zeta)=\log \frac{1}{(1-\bar{z} \zeta)^{2}} \frac{1}{(1-\bar{z} \zeta)^{2}}
$$

\section{Generalization of the result of Bekollé and Bonami}

For a positive Borel measure $\mu$ on $\mathbb{D}$ and an analytic function $\Psi$ in $D(1,1)$ such that its restriction to the interval $(0,2)$ is a real positive function, define

$$
P_{\Psi, \mu}^{+}(f)(z)=\int_{\mathbb{D}}\left|\frac{\Psi(1-\bar{\zeta} z)}{1-\bar{\zeta} z}\right| f(\zeta) d \mu(\zeta), \quad f \in L_{\mu}^{1}, \quad z \in \mathbb{D} .
$$

To obtain a dyadic model for the operator $P_{\Psi, \mu}^{+}$we define the dyadic grids

$$
\mathcal{D}^{\beta}=\left\{I_{j, m}^{\beta}: j \in \mathbb{N} \cup\{0\}, m \in \mathbb{N} \cup\{0\}, 0 \leq m \leq 2^{j}-1\right\}, \quad \beta \in\{0,1 / 2\},
$$

where

$$
I_{j, m}^{\beta}=\left\{e^{i \theta}: \theta \in\left[\frac{4 \pi(m+\beta)}{2^{j}}, \frac{4 \pi(m+1+\beta)}{2^{j}}\right)\right\} .
$$

For each interval $I \subset \mathbb{T}$, with the convention $I=(\alpha, \beta)=\left(\alpha+e^{i 2 \pi j}, \beta+e^{i 2 \pi j}\right)$ for all $j \in \mathbb{N} \cup\{0\}$, there exists $K=K(I) \in \mathcal{D} \cup \mathcal{D}^{1 / 2}$ such that $I \subset K$ and $|K| \leq 4|I|$. Define the positive dyadic kernels

$$
K_{\Psi}^{\beta}(z, \zeta)=\sum_{I \in \mathcal{D}^{\beta}} \frac{1_{S(I)}(z) 1_{S(I)}(\zeta) \Psi(|I|)}{|I|}, \quad z, \zeta \in \mathbb{D}, \quad \beta \in\{0,1 / 2\},
$$


where $S(I)=\left\{r e^{i \theta}: 1-|I| \leq r<1, e^{i \theta} \in I\right\}$ is the Carleson square associated to $I$, and $|I|$ stands for the normalized arc-length of the interval $I$. For this kernel and a positive Borel measure $\mu$ on $\mathbb{D}$, define the dyadic operator

$$
P_{\Psi, \mu}^{\beta}(f)(z)=\sum_{I \in \mathcal{D}^{\beta}}\left\langle f, \frac{1_{S(I)} \Psi(|I|)}{|I|}\right\rangle_{L_{\mu}^{2}} 1_{S(I)}(z), \quad z \in \mathbb{D},
$$

and write

$$
K_{\Psi}(z, \zeta)=\frac{\Psi(|1-\bar{\zeta} z|)}{|1-\bar{\zeta} z|}, \quad z, \zeta \in \mathbb{D}
$$

for short.

The first lemma relates the operator $P_{\Psi, \mu}^{+}$to the sum of the dyadic operators $P_{\Psi, \mu}^{\beta}, \beta \in$ $\{0,1 / 2\}$, by means of a simple pointwise estimate for the inducing kernels.

Lemma 6. Let $\Psi$ be a positive essentially decreasing function on $(0,2)$ such that $\Psi(t) \leq$ $C \Psi(2 t)$ for all $t \in(0,1)$ and for some $C=C(\Psi)>0$. Then there exists a constant $C_{1}=$ $C_{1}(\Psi)>1$ such that

$$
C_{1}^{-1}\left(K_{\Psi}^{0}(z, \zeta)+K_{\Psi}^{1 / 2}(z, \zeta)\right) \leq K_{\Psi}(z, \zeta) \leq C_{1}\left(K_{\Psi}^{0}(z, \zeta)+K_{\Psi}^{1 / 2}(z, \zeta)\right), \quad z, \zeta \in \mathbb{D} .
$$

Proof. Let $\beta \in\{0,1 / 2\}$ and $z, \zeta \in \mathbb{D}$. If both $z$ and $\zeta$ are distinct from zero, choose $I_{0}=$ $I_{0}(z, \zeta) \in \mathcal{D}^{\beta}$ of minimal length such that $\left|I_{0}\right| \geq \max \{1-|z|, 1-|\zeta|\}$ and $z /|z|, \zeta /|\zeta| \in I_{0}$, for otherwise, take $I_{0}=I_{0,0}^{\beta}$. Then $z, \zeta \in S\left(I_{0}\right)$. Let $N \in \mathbb{N}$ such that $2^{N}\left|I_{0}\right|=4$. Since $\Psi$ is essentially decreasing by the hypothesis, we deduce

$$
\begin{aligned}
\sum_{I \in \mathcal{D}^{\beta}} \frac{1_{S(I)}(z) 1_{S(I)}(\zeta) \Psi(|I|)}{|I|} & =\sum_{I \in \mathcal{D}^{\beta}, I_{0} \subset I} \frac{\Psi(|I|)}{|I|}=\sum_{k=0}^{N} \frac{\Psi\left(2^{k}\left|I_{0}\right|\right)}{2^{k}\left|I_{0}\right|} \\
& \lesssim \frac{\Psi\left(\left|I_{0}\right|\right)}{\left|I_{0}\right|} \sum_{k=0}^{N} \frac{1}{2^{k}} \lesssim \frac{\Psi\left(\left|I_{0}\right|\right)}{\left|I_{0}\right|}
\end{aligned}
$$

A direct calculation shows that $|1-\bar{\zeta} z| \leq C\left|I_{0}\right|$ for some $C>1$. As $\Psi$ is essentially decreasing and admits the doubling property, we obtain

$$
\sum_{I \in \mathcal{D}^{\beta}} \frac{1_{S(I)}(z) 1_{S(I)}(\zeta) \Psi(|I|)}{|I|} \lesssim \frac{\Psi\left(\frac{|1-\bar{\zeta} z|}{C}\right)}{|1-\bar{\zeta} z|} \lesssim \frac{\Psi(|1-\bar{\zeta} z|)}{|1-\bar{\zeta} z|}
$$

Since $\beta$ was either 0 or $1 / 2$, the left-hand inequality in (3.6) is proved.

To prove the right hand inequality, let $z, \zeta \in \mathbb{D}$. Let $J=J(z, \zeta) \subset \mathbb{T}$ such that $z, \zeta \in S(J)$ and $|J| \asymp|1-\bar{\zeta} z|$, see [2] for details. There exist $\beta \in\{0,1 / 2\}$ and $K \in \mathcal{D}^{\beta}$ such that $J \subset K$ and $|K| \leq 4|J|$. By using the hypotheses on $\Psi$, we get

$$
\frac{\Psi(|1-\bar{\zeta} z|)}{|1-\bar{\zeta} z|} \lesssim \frac{\Psi(|J|)}{|J|} \lesssim \frac{\Psi(|K|)}{|K|} \lesssim \sum_{\substack{I \in \mathcal{D}^{\beta} \\ K \subset J}} \frac{1_{S(I)}(z) 1_{S(I)}(\zeta) \Psi(|I|)}{|I|} \lesssim K_{\Psi}^{0}(z, \zeta)+K_{\Psi}^{1 / 2}(z, \zeta),
$$

and the lemma is proved.

For a positive Borel measure $\nu$ and a dyadic grid $\mathcal{D}$ on $\mathbb{T}$, the dyadic weighted HardyLittlewood (or Hörmander type) maximal function is defined as

$$
M_{\nu, \mathcal{D}}(f)(z)=\sup _{I \in \mathcal{D}} \frac{1_{S(I)}(z)}{\nu(S(I))} \int_{S(I)}|f(\zeta)| d \nu(\zeta) .
$$

The maximal operator $M_{\nu, \mathcal{D}^{\beta}}$ appears naturally in the study of the dyadic operator $P_{\Psi, \mu}^{\beta}$. Its standard boundedness properties are given in the next lemma. 
Lemma A. Let $\nu$ be a positive Borel measure and $\mathcal{D}$ a dyadic grid on $\mathbb{T}$. Then $M_{\nu, \mathcal{D}}: L_{\nu}^{1} \rightarrow$ $L_{\nu}^{1^{\infty}}$ is bounded and consequently, $M_{\nu, \mathcal{D}}: L_{\nu}^{p} \rightarrow L_{\nu}^{p}$ is bounded for each $1<p \leq \infty$. In particular, there exists a constant $C=C(p)>0$ such that

$$
\left\|M_{\nu, \mathcal{D}}(f)\right\|_{L_{\nu}^{p}} \leq C\|f\|_{L_{\nu}^{p}}
$$

Proof. By the Marcinkiewicz interpolation theorem it is enough to prove the weak $(1,1)$ inequality. Let $f \geq 0, \alpha>0$ and $O_{\alpha}=\left\{z \in \mathbb{D}: M_{\nu, \mathcal{D}} f(z)>\alpha\right\}$. Further, let $\Phi$ be the family of Carleson squares $S \in \mathcal{D}$ such that

$$
\int_{S}|f(\zeta)| d \nu(\zeta)>\alpha \nu(S)
$$

and let $\Phi^{\max }$ the subfamily of $\Phi$ consisting of the maximal Carleson squares. Then $\Phi^{\max }$ is a covering of $O_{\alpha}$ and each $z \in O_{\alpha}$ is contained in at most two different squares in $\Phi^{\max }$. Therefore

$$
\nu\left(O_{\alpha}\right) \leq \sum_{S \in \Phi^{\max }} \nu(S) \leq \frac{1}{\alpha} \sum_{S \in \Phi^{\max }} \int_{S}|f(\zeta)| d \nu(\zeta) \leq \frac{2}{\alpha} \int_{O_{\alpha}}|f(\zeta)| d \nu(\zeta) \leq \frac{2}{\alpha}\|f\|_{L_{\nu}^{1}},
$$

and the lemma is proved.

Let $v, u \in L_{\mu}^{1}$ non-negative, and let $1<p<\infty$ and $p^{\prime}$ its dual exponent. The dual weight of $v$ is $\sigma=\sigma(p, v)=v^{1-p^{\prime}}$. If $T$ is a linear operator, the following are equivalent:

(A) $T: L_{\mu}^{p}(v) \rightarrow L_{\mu}^{p}(u)$ is bounded;

(B) $T(\sigma \cdot): L_{\mu}^{p}(\sigma) \rightarrow L_{\mu}^{p}(u)$ is bounded;

(C) $u^{1 / p} T\left(\sigma^{1 / p^{\prime}}.\right): L_{\mu}^{p} \rightarrow L_{\mu}^{p}$ bounded.

Moreover,

$$
\|T\|_{L_{\mu}^{p}(v) \rightarrow L_{\mu}^{p}(u)}=\|T(\sigma \cdot)\|_{L_{\mu}^{p}(\sigma) \rightarrow L_{\mu}^{p}(u)}=\left\|u^{1 / p} T\left(\sigma^{1 / p^{\prime}} \cdot\right)\right\|_{L_{\mu}^{p} \rightarrow L_{\mu}^{p}} .
$$

We now show how to obtain a linear bound for our dyadic operator in terms of the $B_{p, \mu^{-}}$ characteristic. This requires some hypotheses on the measure $\mu$ and the function $\Psi$. The following theorem is an extension of the main result of [15].

Theorem 7. Let $1<p<\infty, \mu$ a positive Borel measure on $\mathbb{D}$ and $v \in B_{p, \mu}$. Let $\Psi$ : $D(1,1) \rightarrow \mathbb{C}$ be an analytic function such that its restriction to the interval $(0,2)$ is positive and $|\Psi(1-z)|=|\Psi(1-\bar{z})|$ for all $z \in \mathbb{D}$. Further, assume that $\mu(S(I)) \lesssim \mu(T(I))$ and $\Psi(|I|) \mu(S(I)) \lesssim|I|$ for all dyadic intervals $I$. Then

$$
\left\|P_{\Psi, \mu}^{\beta}(f)\right\|_{L_{\mu}^{p}(v)} \lesssim B_{p, \mu}(v)^{\max \left\{1, \frac{1}{p-1}\right\}}\|f\|_{L_{\mu}^{p}(v)}, \quad \beta \in\left\{0, \frac{1}{2}\right\} .
$$

Proof. We focus first on the case $p=2$ since it is easiest. We then explain how to either obtain the result for all $p$ from this or how to modify the proof given to provide a direct proof for all $p$.

We will proceed by duality to study the norm of $P_{\Psi, \mu}^{\beta}\left(v^{-1} \cdot\right): L_{\mu}^{2}\left(v^{-1}\right) \rightarrow L_{\mu}^{2}(v)$. Then the assertion for $p=2$ follows by (3.10). Suppose that $f \in L_{\mu}^{2}\left(v^{-1}\right)$ and $g \in L_{\mu}^{2}(v)$ are 
non-negative functions. The role of $\beta$ now plays no role and so we drop its dependence. Then

$$
\begin{aligned}
& \int_{\mathbb{D}} P_{\Psi, \mu}\left(v^{-1} f\right)(z) g(z) v(z) d \mu(z) \\
& =\sum_{I \in \mathcal{D}}\left\langle v^{-1} f, 1_{S(I)}\right\rangle_{L_{\mu}^{2}}\left\langle v g, 1_{S(I)}\right\rangle_{L_{\mu}^{2}} \frac{\Psi(|I|)}{|I|} \\
& =\sum_{I \in \mathcal{D}} \frac{\Psi(|I|) \mu(S(I))^{2}}{|I|}\left(\frac{\int_{S(I)} f v^{-1} d \mu}{\int_{S(I)} v^{-1} d \mu}\right)\left(\frac{\int_{S(I)} g v d \mu}{\int_{S(I)} v d \mu}\right) \frac{\int_{S(I)} v^{-1} d \mu}{\mu(S(I))} \frac{\int_{S(I)} v d \mu}{\mu(S(I))} \\
& \lesssim B_{2, \mu}(v) \sum_{I \in \mathcal{D}} \mu(T(I))\left(\frac{\int_{S(I)} f v^{-1} d \mu}{\int_{S(I)} v^{-1} d \mu}\right)\left(\frac{\int_{S(I)} g v d \mu}{\int_{S(I)} v d \mu}\right) \\
& =B_{2, \mu}(v) \sum_{I \in \mathcal{D}} \int_{T(I)}\left(\frac{\int_{S(I)} f v^{-1} d \mu}{\int_{S(I)} v^{-1} d \mu}\right)\left(\frac{\int_{S(I)} g v d \mu}{\int_{S(I)} v d \mu}\right) d \mu(z) \\
& \leq B_{2, \mu}(v) \int_{\mathbb{D}}\left(M_{v^{-1} \mu, \mathcal{D}}(f)(z) v^{-\frac{1}{2}}(z)\right)\left(M_{v \mu, \mathcal{D}}(g)(z) v^{\frac{1}{2}}(z)\right) d \mu(z) \\
& \lesssim B_{2, \mu}(v)\|f\|_{L_{\mu}^{2}\left(v^{-1}\right)}\|g\|_{L_{\mu}^{2}(v)},
\end{aligned}
$$

where the first inequality follows from the hypotheses on $\mu, \Psi$ and $v$; the second by the domination of the averages by the maximal functions; and the last by the Cauchy-Schwarz inequality and the boundedness of the maximal functions due to Lemma A.

It is possible to use the standard extrapolation proof to show that this estimate can be lifted to $1<p<\infty$ with an appropriate change in the characteristic for the weight $v$; see [15] for these details. It is instead possible to provide a direct proof by using a verbatim repetition of the proof above. We sketch the modifications now and leave the details to the reader.

Consider first the case $1<p \leq 2$. Let $\sigma=v^{1-p^{\prime}}$. The goal is to now prove that

$$
\left\|P_{\Psi, \mu}(\sigma f)\right\|_{L_{\mu}^{p}(v)} \lesssim B_{p, \mu}(v)^{\frac{1}{p-1}}\|f\|_{L_{\mu}^{p}(\sigma)} .
$$

It is more convenient to prove the equivalent inequality

$$
\left\|P_{\Psi, \mu}(\sigma f)^{p-1}\right\|_{L_{\mu}^{p^{\prime}(v)}} \lesssim B_{p, \mu}(v)\|f\|_{L_{\mu}^{p}(\sigma)}^{p-1} .
$$

This last inequality can be studied via duality as above. Since $1<p \leq 2$, and the function $h(x)=x^{r}$ is sub-additive for $0<r<1$ we obtain

$$
\begin{aligned}
\left\langle P_{\Psi, \mu}(\sigma f)^{p-1}, v g\right\rangle_{L_{\mu}^{2}} & \leq \sum_{I \in \mathcal{D}}\left\langle\sigma f, 1_{S(I)}\right\rangle_{L_{\mu}^{2}}^{p-1}\left(\frac{\Psi(|I|)}{|I|}\right)^{p-1}\left\langle v g, 1_{S(I)}\right\rangle_{L_{\mu}^{2}} \\
& \lesssim B_{p, \mu}(v)\left\|M_{\sigma \mu}(f)\right\|_{L_{\mu}^{p}(\sigma)}^{p-1}\left\|M_{v \mu}(g)\right\|_{L_{\mu}^{p}(v)} .
\end{aligned}
$$

The inequality above is obtained exactly as above in the case $p=2$ by using the definition of $B_{p, \mu}(v)$, the relationship between $\mu$ and $\Psi$. Estimates of the maximal function then provide the desired estimates to control the duality. The case $2<p<\infty$ can be deduced via the self-adjointness of $P_{\Psi, \mu}$ with respect to $\langle\cdot, \cdot\rangle_{L_{\mu}^{2}}$, the result for $1<p<2$ and the relationship between $B_{p, \mu}(v)$ and $B_{p^{\prime}, \mu}(v)$.

Because of the equivalence we have between the dyadic operators $P_{\Psi, \mu}^{\beta}$ and and $P_{\Psi, \mu}^{+}$given in Lemma 6, we obtain the following result.

Corollary 8. Let $1<p<\infty, \mu$ a positive Borel measure on $\mathbb{D}$ and $v \in B_{p, \mu}$. Let $\Psi$ : $D(1,1) \rightarrow \mathbb{C}$ be an analytic function such that its restriction to the interval $(0,2)$ is positive and essentially decreasing, $\Psi(t) \lesssim \Psi(2 t)$ for all $t \in(0,1)$, and $|\Psi(1-z)|=|\Psi(1-\bar{z})|$ for all $z \in \mathbb{D}$. Further, assume that $\mu(S(I)) \lesssim \mu(T(I))$ and $\Psi(|I|) \mu(S(I)) \lesssim|I|$ for all intervals $I$. 
Then

$$
\left\|P_{\Psi, \mu}^{+}\right\|_{L_{\mu}^{p}(v) \rightarrow L_{\mu}^{p}(v)} \lesssim B_{p, \mu}(v)^{\max \left\{1, \frac{1}{p-1}\right\}} .
$$

The upper bound for the operator norm given in Corollary 8 is essentially independent of $\Psi$, and therefore it is not necessarily sharp for all admissible $\Psi$. But when we apply it in the proof of Theorem 2 to deduce that $v \in B_{p, \omega}$ is a sufficient condition for $P_{\omega}^{+}: L_{\omega}^{p}(v) \rightarrow L_{\omega}^{p}(v)$ to be bounded, the hypotheses on $\Psi$ and $\omega$ in question are satisfied precisely, meaning that $\lesssim$ are in fact $\asymp$, and hence the resulting sufficient condition will also be necessary. This will be discussed in more detail at the end of the section when the proof of Theorem 2 is finally pulled together.

We next proceed with auxiliary results needed to show that $v \in B_{p, \omega}$ is a necessary condition for $P_{\omega}: L_{\omega}^{p}(v) \rightarrow L_{\omega}^{p, \infty}(v)$ to be bounded.

Lemma 9. Let $\omega$ be a positive Borel measure such that $B_{z}^{\omega}$ admits the representation

$$
B_{z}^{\omega}(\zeta)=\frac{1}{(1-\bar{z} \zeta)^{\gamma}} \int_{0}^{1} \frac{d \nu(r)}{1-r \bar{z} \zeta}, \quad z, \zeta \in \mathbb{D}
$$

for some $\gamma \geq 1$ and a positive measure $\nu$ supported on $[0,1]$, and let $c>1$. Then

$$
\left|B_{z_{0}}^{\omega}(\zeta)-B_{z}^{\omega}(\zeta)\right| \leq C \frac{\left|z-z_{0}\right|}{|1-\bar{\zeta} z|}\left|B_{z}^{\omega}(\zeta)\right|
$$

for all $z, z_{0}, \zeta \in \mathbb{D}$ with $|1-\bar{\zeta} z| \geq c\left|z-z_{0}\right|$, where

$$
C=C(c, \gamma)=\sqrt{2}(2+\gamma) \frac{c^{\gamma+1}(3 c+1)}{(c-1)^{\gamma+2}} \rightarrow 3 \sqrt{2}(2+\gamma), \quad c \rightarrow \infty .
$$

Proof. A direct calculation shows that

$$
\left|B_{z_{0}}^{\omega}(\zeta)-B_{z}^{\omega}(\zeta)\right|=\left|B_{\zeta}^{\omega}\left(z_{0}\right)-B_{\zeta}^{\omega}(z)\right|=\left|\int_{z_{0}}^{z}\left(B_{\zeta}^{\omega}\right)^{\prime}(x) d x\right| \leq \int_{z_{0}}^{z}\left|\left(B_{\zeta}^{\omega}\right)^{\prime}(x)\right||d x|,
$$

where

$$
B_{\zeta}^{\prime}(x)=\frac{\gamma \bar{\zeta}}{(1-\bar{\zeta} x)^{\gamma+1}} \int_{0}^{1} \frac{d \nu(r)}{1-r \bar{\zeta} x}+\frac{\bar{\zeta}}{(1-\bar{\zeta} x)^{\gamma}} \int_{0}^{1} \frac{r d \nu(r)}{(1-r \bar{\zeta} x)^{2}}, \quad \zeta, x \in \mathbb{D}
$$

and hence

$$
\left|B_{\zeta}^{\prime}(x)\right| \leq \frac{\gamma|\zeta|}{|1-\bar{\zeta} x|^{\gamma+1}} \int_{0}^{1} \frac{d \nu(r)}{|1-r \bar{\zeta} x|}+\frac{|\zeta|}{|1-\bar{\zeta} x|^{\gamma}} \int_{0}^{1} \frac{d \nu(r)}{|1-r \bar{\zeta} x|^{2}}, \quad \zeta, x \in \mathbb{D} .
$$

Since $|1-w| \leq 2|1-r w|$ for all $w \in \mathbb{D}$ and $0 \leq r \leq 1$, we deduce

It follows that

$$
\left|B_{\zeta}^{\prime}(x)\right| \leq \frac{(2+\gamma)|\zeta|}{|1-\bar{\zeta} x|^{\gamma+1}} \int_{0}^{1} \frac{d \nu(r)}{|1-r \bar{\zeta} x|}, \quad \zeta, x \in \mathbb{D} .
$$

$$
\left|B_{z_{0}}^{\omega}(\zeta)-B_{z}^{\omega}(\zeta)\right| \leq(2+\gamma)|\zeta|\left|z-z_{0}\right| \sup _{x \in\left[z, z_{0}\right]}\left(\frac{1}{|1-\bar{\zeta} x|^{\gamma+1}} \int_{0}^{1} \frac{d \nu(r)}{|1-r \bar{\zeta} x|}\right) .
$$

If $x \in\left[z, z_{0}\right]$, then $|1-\bar{\zeta} z| \geq c\left|z-z_{0}\right| \geq c|z-x|$, and hence

$$
|1-\bar{\zeta} x|=|1-\bar{\zeta} z+\bar{\zeta} z-\bar{\zeta} x| \geq|1-\bar{\zeta} z|-|\zeta||z-x| \geq|1-\bar{\zeta} z|-c^{-1}|1-\bar{\zeta} z|=\left(1-\frac{1}{c}\right)|1-\bar{\zeta} z| .
$$

Thus

Let $\delta \in(0,1)$. Then

$$
\left|B_{z_{0}}^{\omega}(\zeta)-B_{z}^{\omega}(\zeta)\right| \leq \frac{(2+\gamma)|\zeta|\left|z-z_{0}\right|}{\left(1-\frac{1}{c}\right)^{\gamma+1}|1-\bar{\zeta} z|^{\gamma+1}} \sup _{x \in\left[z, z_{0}\right]}\left(\int_{0}^{1} \frac{d \nu(r)}{|1-r \bar{\zeta} x|}\right)
$$

$$
\int_{0}^{1} \frac{d \nu(r)}{|1-r \bar{\zeta} x|} \leq \frac{1}{1-\delta} \nu([0,1])+\int_{\delta}^{1} \frac{d \nu(r)}{|1-r \bar{\zeta} x|} \leq \frac{2}{1-\delta} \int_{0}^{1} \frac{d \nu(r)}{|1-r \bar{\zeta} z|}+\int_{\delta}^{1} \frac{d \nu(r)}{|1-r \bar{\zeta} x|}
$$


A direct calculation or a geometric reasoning shows that $|1-w| \leq \frac{2}{1+\delta}|1-r w|$ for all $w \in \mathbb{D}$ and $\delta \leq r \leq 1$. Hence

$$
|1-r \bar{\zeta} x| \geq|1-r \bar{\zeta} z|-c^{-1}|1-\bar{\zeta} z| \geq|1-r \bar{\zeta} z|-\frac{2}{c(1+\delta)}|1-r \bar{\zeta} z|, \quad \delta \leq r \leq 1 .
$$

By choosing $\delta=1 / c$, we deduce

$$
|1-r \bar{\zeta} x| \geq \frac{c-1}{c+1}|1-r \bar{\zeta} z|
$$

and it follows that

$$
\int_{0}^{1} \frac{d \nu(r)}{|1-r \bar{\zeta} x|} \leq \frac{3 c+1}{c-1} \int_{0}^{1} \frac{d \nu(r)}{|1-r \bar{\zeta} z|}
$$

Since

$$
\begin{aligned}
\left|\int_{0}^{1} \frac{d \nu(r)}{1-r z}\right| & =\left(\left(\int_{0}^{1} \frac{(1-r|z| \cos (\theta)) d \nu(r)}{|1-r z|^{2}}\right)^{2}+\left(\int_{0}^{1} \frac{r|z| \sin (\theta) d \nu(r)}{|1-r z|^{2}}\right)^{2}\right)^{1 / 2} \\
& \geq \frac{1}{\sqrt{2}} \int_{0}^{1} \frac{[(1-r|z| \cos (\theta))+r|z||\sin (\theta)|] d \nu(r)}{|1-r z|^{2}} \\
& \geq \frac{1}{\sqrt{2}} \int_{0}^{1} \frac{d \nu(r)}{|1-r z|}, \quad z \in \mathbb{D}
\end{aligned}
$$

we deduce

$$
\begin{aligned}
\left|B_{z_{0}}^{\omega}(\zeta)-B_{z}^{\omega}(\zeta)\right| & \leq \frac{(2+\gamma)|\zeta|\left|z-z_{0}\right|}{\left(1-\frac{1}{c}\right)^{\gamma+1}|1-\bar{\zeta} z|^{\gamma+1}} \frac{3 c+1}{c-1} \int_{0}^{1} \frac{d \nu(r)}{|1-r \bar{\zeta} z|} \\
& \leq \frac{\sqrt{2}(2+\gamma)|\zeta|\left|z-z_{0}\right|}{\left(1-\frac{1}{c}\right)^{\gamma+1}|1-\bar{\zeta} z|^{\gamma+1}} \frac{3 c+1}{c-1}\left|\int_{0}^{1} \frac{d \nu(r)}{1-r \bar{\zeta} z \mid}\right| \leq C \frac{\left|z-z_{0}\right|}{|1-\bar{\zeta} z|}\left|B_{z}^{\omega}(\zeta)\right|
\end{aligned}
$$

where $C=C(c, \gamma)=\sqrt{2}(2+\gamma) \frac{c^{\gamma+1}(3 c+1)}{(c-1)^{\gamma+2}}$.

Lemma 10. Let $\omega \in \widehat{\mathcal{D}}$ such that $B_{z}^{\omega}$ admits the representation

$$
B_{z}^{\omega}(\zeta)=\frac{1}{(1-\bar{z} \zeta)^{\gamma}} \int_{0}^{1} \frac{d \nu(r)}{1-r \bar{z} \zeta}, \quad z, \zeta \in \mathbb{D}
$$

for some $\gamma \geq 1$ and a positive measure $\nu$ supported on $[0,1]$. Then

$$
\int_{0}^{1} \frac{d \nu(r)}{1-r x} \asymp \frac{(1-x)^{\gamma-1}}{\widehat{\omega}(x)}, \quad x \in[0,1) .
$$

Proof. By [14, Lemmas 3.1 and 3.2], see also [11, Lemma 6.2],

$$
\frac{1}{\left(1-|z|^{2}\right) \widehat{\omega}\left(|z|^{2}\right)} \asymp\left\|B_{z}^{\omega}\right\|_{A_{\omega}^{2}}^{2}=B_{z}^{\omega}(z)=\frac{1}{\left(1-|z|^{2}\right)^{\gamma}} \int_{0}^{1} \frac{d \nu(r)}{1-r|z|^{2}}, \quad z \in \mathbb{D},
$$

which is equivalent to (3.13).

For a Carleson square $S=S(I)$, let $\ell(S)=|I|$ denote its side length.

Lemma 11. Let $\omega \in \widehat{\mathcal{D}}$ such that $B_{z}^{\omega}$ admits the representation (3.12) for some $\gamma \geq 1$ and a positive measure $\nu$ supported on $[0,1]$. Then there are constants $D_{1}=D_{1}(\gamma)>0$ and $D_{2}=D_{2}(\gamma)>0$ such that for all (sufficiently small) Carleson squares $S_{1}$ and $S_{2}$, with $\ell\left(S_{1}\right)=\ell\left(S_{2}\right)$ and $D_{1} \ell\left(S_{1}\right) \leq \operatorname{dist}\left(S_{1}, S_{2}\right) \leq D_{2} \ell\left(S_{1}\right)$, we have

$$
\left|P_{\omega}(f)(z)\right| \geq C \frac{\int_{S_{1}} f(\zeta) \omega(\zeta) d A(\zeta)}{\omega\left(S_{1}\right)}, \quad z \in S_{2},
$$

for some constant $C=C\left(D_{1}, D_{2}, \omega\right)>0$ and for all nonnegative functions $f$ supported on $S_{1}$. 
Proof. Let $S_{1}$ and $S_{2}$ be (small) Carleson squares such that $\ell\left(S_{1}\right)=\ell\left(S_{2}\right)$ and $D_{1} \ell\left(S_{1}\right) \leq$ $\operatorname{dist}\left(S_{1}, S_{2}\right) \leq D_{2} \ell\left(S_{1}\right)$, where $D_{1}, D_{2}>0$ are absolute constants to be fixed later. Let $\zeta_{0}$ be the center of $S_{1}$. Then

$$
\left|P_{\omega}(f)(z)\right| \geq\left|B_{\zeta_{0}}^{\omega}(z)\right| \int_{S_{1}} f(\zeta) \omega(\zeta) d A(\zeta)-\int_{S_{1}} f(\zeta)\left|B_{\zeta}^{\omega}(z)-B_{\zeta_{0}}^{\omega}(z)\right| \omega(\zeta) d A(\zeta)
$$

for all $z \in \mathbb{D}$. If $z \in S_{2}$ and $\zeta \in S_{1}$, then

$$
\left|1-\bar{z} \zeta_{0}\right| \geq\left|z-\zeta_{0}\right| \geq \frac{\ell\left(S_{1}\right)}{3}+\operatorname{dist}\left(S_{1}, S_{2}\right) \geq\left(\frac{1}{3}+D_{1}\right) \ell\left(S_{1}\right) \geq c_{1}\left|\zeta-\zeta_{0}\right|,
$$

where $c_{1}=\frac{\left(\frac{1}{3}+D_{1}\right)}{\sqrt{2}}$. Choose $D_{1}=D_{1}(\gamma)>1$ sufficiently large such that $c_{1}>1$ and $\sqrt{2}(2+$ $\gamma) \frac{c_{1}^{\gamma}\left(3 c_{1}+1\right)}{\left(c_{1}-1\right)^{\gamma+2}} \leq \frac{1}{2}$. Then, by using Lemma 9 and (3.16), we deduce

$$
\begin{aligned}
\left|B_{\zeta}^{\omega}(z)-B_{\zeta_{0}}^{\omega}(z)\right| & \leq \sqrt{2}(2+\gamma) \frac{c_{1}^{\gamma+1}\left(3 c_{1}+1\right)}{\left(c_{1}-1\right)^{\gamma+2}} \frac{\left|\zeta_{0}-\zeta\right|}{\left|1-\zeta_{0} \bar{z}\right|}\left|B_{\zeta_{0}}^{\omega}(z)\right| \\
& \leq \sqrt{2}(2+\gamma) \frac{c_{1}^{\gamma}\left(3 c_{1}+1\right)}{\left(c_{1}-1\right)^{\gamma+2}}\left|B_{\zeta_{0}}^{\omega}(z)\right| \leq \frac{1}{2}\left|B_{\zeta_{0}}^{\omega}(z)\right| .
\end{aligned}
$$

By combining (3.15) and (3.17) we get

$$
\left|P_{\omega}(f)(z)\right| \geq \frac{1}{2}\left|B_{\zeta_{0}}^{\omega}(z)\right| \int_{S_{1}} f(\zeta) \omega(\zeta) d A(\zeta), \quad z \in S_{2}
$$

Now, we observe that

$$
\left.\left|1-\bar{\zeta}_{0} z\right| \leq\left(1-\left|\zeta_{0}\right|^{2}\right)+\left|\zeta_{0}-z\right|\right) \leq 3 \ell\left(S_{1}\right)+\operatorname{dist}\left(S_{1}, S_{2}\right) \leq\left(3+D_{2}\right) \ell\left(S_{1}\right), \quad z \in S_{2}
$$

This together with (3.11), the inequality $(a+x b) \leq x(a+b)$ for $a, b>0$ and $x \geq 1$, and Lemma 10 yield

$$
\begin{aligned}
\left|B_{\zeta_{0}}^{\omega}(z)\right| & \geq \frac{1}{\sqrt{2}\left|1-\bar{\zeta}_{0} z\right|^{\gamma}} \int_{0}^{1} \frac{d \nu(r)}{1-r\left(1-\left|1-\bar{\zeta}_{0} z\right|\right)} \\
& \geq \frac{1}{\sqrt{2}\left(3+D_{2}\right)^{\gamma} \ell\left(S_{1}\right)^{\gamma}} \int_{0}^{1} \frac{d \nu(r)}{1-r+r\left(3+D_{2}\right) \ell\left(S_{1}\right)} \\
& \geq \frac{1}{\sqrt{2}\left(3+D_{2}\right)^{\gamma+1} \ell\left(S_{1}\right)^{\gamma}} \int_{0}^{1} \frac{d \nu(r)}{1-r+r \ell\left(S_{1}\right)} \geq \frac{C}{\sqrt{2}\left(3+D_{2}\right)^{\gamma+1} \omega\left(S_{1}\right)}
\end{aligned}
$$

for some constant $C=C(\omega)>0$. The assertion follows by combining this with (3.18).

Proposition 12. Let $1<p<\infty, \omega \in \widehat{\mathcal{D}}$ such that $B_{z}^{\omega}$ admits the representation (3.12) for some $\gamma \geq 1$ and a positive measure $\nu$ supported on $[0,1]$, and $v \in L_{\omega, \text { loc }}^{1}$ non-negative. If $P_{\omega}: L_{\omega}^{p}(v) \rightarrow L_{\omega}^{p, \infty}(v)$ is bounded, then $v \in B_{p, \omega}$.

Proof. It suffices to show that the quantity

$$
\frac{(v \omega)(S)}{\omega(S)}\left(\frac{\left(v^{-\frac{p^{\prime}}{p}} \omega\right)(S)}{\omega(S)}\right)^{\frac{p}{p^{\prime}}}
$$

is uniformly bounded for all small Carleson squares $S$. By the hypothesis, there exists $C_{1}>0$ such that

$$
\lambda^{p}(v \omega)\left(\left\{z \in \mathbb{D}:\left|P_{\omega}(f)(z)\right| \geq \lambda\right\}\right) \leq C_{1}\|f\|_{L_{\omega}^{p}(v)}^{p}, \quad \lambda>0 .
$$

Let $S_{1}$ be a sufficiently small Carleson square, and choose

$$
\lambda=C \frac{\int_{S_{1}}\left(\min \left\{n, v^{-\frac{p^{\prime}}{p}}(\zeta)\right\}\right) d(\omega \otimes m)(\zeta)}{\omega\left(S_{1}\right)}, \quad n \in \mathbb{N},
$$


where $C$ is the constant appearing in (3.14). Further, choose $f=1_{S_{1}} \min \left\{n, v^{-\frac{p^{\prime}}{p}}\right\}$. Then we get

$$
\begin{aligned}
& \frac{\left(\int_{S_{1}} \min \left\{n, v^{-\frac{p^{\prime}}{p}}(\zeta)\right\} d(\omega \otimes m)(\zeta)\right)^{p-1}}{\omega\left(S_{1}\right)^{p}} \\
& \cdot(v \omega)\left(\left\{z \in \mathbb{D}:\left|P_{\omega}(f)(z)\right| \geq C \frac{\int_{S_{1}} \min \left\{n, v^{-\frac{p^{\prime}}{p}}(\zeta)\right\} d(\omega \otimes m)(\zeta)}{\omega\left(S_{1}\right)}\right\}\right) \leq \frac{C_{1}}{C^{p}} .
\end{aligned}
$$

By Lemma 11, for all suitable $S_{2}$ with $\ell\left(S_{2}\right)=\ell\left(S_{1}\right)$ we have

$$
S_{2} \subset\left\{z \in \mathbb{D}:\left|P_{\omega}(f)(z)\right| \geq C \frac{\int_{S_{1}} \min \left\{n, v^{-\frac{p^{\prime}}{p}}(\zeta)\right\} d(\omega \otimes m)(\zeta)}{\omega\left(S_{1}\right)}\right\},
$$

and it follows that

$$
\frac{\left(\int_{S_{1}} \min \left\{n, v^{-\frac{p^{\prime}}{p}}(\zeta)\right\} d(\omega \otimes m)(\zeta)\right)^{p-1} \int_{S_{2}} v(\zeta) d(\omega \otimes m)(\zeta)}{\omega\left(S_{1}\right)^{p}} \leq \frac{C_{1}}{C^{p}} .
$$

By changing the roles of $S_{1}$ and $S_{2}$ we deduce

$$
\frac{\left(\int_{S_{2}} \min \left\{n, v^{-\frac{p^{\prime}}{p}}(\zeta)\right\} d(\omega \otimes m)(\zeta)\right)^{p-1} \int_{S_{1}} v(\zeta) d(\omega \otimes m)(\zeta)}{\omega\left(S_{2}\right)^{p}} \leq \frac{C_{1}}{C^{p}},
$$

and it follows that $v \in L_{\omega}^{1}$. By letting $n \rightarrow \infty$ and using Fatou's lemma we deduce

$$
\frac{\left(\int_{S_{1}} v^{-\frac{p^{\prime}}{p}} d(\omega \otimes m)\right)^{p-1} \int_{S_{2}} v d(\omega \otimes m)}{\omega\left(S_{1}\right)^{p}} \frac{\left(\int_{S_{2}} v^{-\frac{p^{\prime}}{p}} d(\omega \otimes m)\right)^{p-1} \int_{S_{1}} v d(\omega \otimes m)}{\omega\left(S_{2}\right)^{p}} \leq \frac{C_{1}^{2}}{C^{2 p}} .
$$

Since

$$
\frac{(v \omega)(S)}{\omega(S)^{p}}\left(\left(v^{-\frac{p^{\prime}}{p}} \omega\right)(S)\right)^{\frac{p}{p^{\prime}}}=\frac{(v \omega)(S)}{\omega(S)}\left(\frac{\left(v^{-\frac{p^{\prime}}{p}} \omega\right)(S)}{\omega(S)}\right)^{\frac{p}{p^{\prime}}} \geq 1
$$

for any Carleson square $S$ by Hölder's inequality, it follows that $v \in B_{p, \omega}$.

With these preparations we are ready to prove the first of our main results.

Proof of Theorem [2. Clearly, (i) $\Rightarrow($ ii) $\Rightarrow$ (iii), and (iii) $\Rightarrow$ (iv) follows by Proposition 12, To see the remaining implication, note that

$$
B_{z}^{\omega}(\zeta)=\frac{1}{(1-\bar{z} \zeta)^{\gamma}} \int_{0}^{1} \frac{d \nu(r)}{1-r \bar{z} \zeta}=\frac{\Psi(1-\bar{z} \zeta)}{1-\bar{z} \zeta}
$$

for the analytic function

$$
\Psi(z)=z^{1-\gamma} \int_{0}^{1} \frac{d \nu(r)}{1-r(1-z)}, \quad z \in D(1,1) .
$$

The restriction of $\Psi$ to $(0,2)$ is decreasing because $\gamma \geq 1$, and obviously $|\Psi(1-z)|=|\Psi(1-\bar{z})|$ for all $z \in \mathbb{D}$. Moreover, $\mu=\omega \otimes m$ satisfies $\mu(S(I)) \lesssim \mu(T(I))$ because $\omega \in \mathcal{R}$, and Lemma 10 yields

$$
\Psi(|I|)=\frac{1}{|I|^{\gamma-1}} \int_{0}^{1} \frac{d \nu(r)}{1-r(1-|I|)} \asymp \frac{1}{\widehat{\omega}(1-|I|)},
$$


so $\Psi(|I|) \mu(S(I)) \asymp|I|$ for all intervals $I$. Now that $\Psi(t) \lesssim \Psi(2 t)$ for all $t \in(0,1)$, the hypothesis of Corollary 8 are satisfied, and hence (iv) $\Rightarrow$ (i) as well as the estimate for the operator norm of $P_{\omega}^{+}$follow.

\section{Weak type $(1,1)$ InEQUality}

Lemma 13. Let $\nu$ be a positive Borel measure supported on $[0,1]$. Then

$$
\left|\int_{0}^{1} \frac{d \nu(r)}{1-r z}\right| \asymp \int_{0}^{1} \frac{d \nu(r)}{|1-r z|} \asymp \int_{0}^{1} \frac{d \nu(r)}{1-r(1-|1-z|)}, \quad z \in \mathbb{D} .
$$

Proof. We first show that

$$
1-r(1-|1-z|) \asymp|1-r z|, \quad z \in \mathbb{D} .
$$

On one hand, $|1-r z|=|1-r+r(1-z)| \leq(1-r)+r|1-z|$ for all $0<r<1$. On the other hand, if $z=|z| e^{i \theta}$ and $r \geq 1 / 2$, then

$$
\begin{aligned}
|1-r z|^{2} & =((1-r)+r(1-|z|))^{2}+4 r|z| \sin ^{2}\left(\frac{\theta}{2}\right) \\
& \geq \frac{1}{4}\left((1-r)^{2}+(1-|z|)^{2}\right)+4 r|z| \sin ^{2}\left(\frac{\theta}{2}\right) \\
& \geq \frac{1}{4}\left((1-r)^{2}+(1-|z|)^{2}+4|z| \sin ^{2}\left(\frac{\theta}{2}\right)\right) \\
& =\frac{1}{4}\left((1-r)^{2}+|1-z|^{2}\right),
\end{aligned}
$$

and hence

$$
|1-r z| \geq \frac{1}{2 \sqrt{2}}((1-r)+|1-z|) .
$$

Moreover, for $0 \leq r \leq 1 / 2$ we have

$$
|1-r z| \geq \frac{1}{2} \geq \frac{((1-r)+|1-z|)}{6},
$$

and hence (4.1) follows. Therefore

$$
\left|\int_{0}^{1} \frac{d \nu(r)}{1-r z}\right| \leq \int_{0}^{1} \frac{d \nu(r)}{|1-r z|} \asymp \int_{0}^{1} \frac{d \nu(r)}{1-r(1-|1-z|)}, \quad z \in \mathbb{D} .
$$

By combining this with (3.11) we deduce the assertion.

Lemma 14. Let $\omega \in \widehat{\mathcal{D}}$ such that $B_{z}^{\omega}$ admits the representation

$$
B_{z}^{\omega}(\zeta)=\frac{1}{(1-\bar{z} \zeta)^{\gamma}} \int_{0}^{1} \frac{d \nu(r)}{1-r \bar{z} \zeta}, \quad z, \zeta \in \mathbb{D}
$$

for some $\gamma \geq 1$ and a positive measure $\nu$ supported on $[0,1]$. Then for $v \in L_{\omega}^{1}$ non-negative, $z_{0} \in \mathbb{D} \backslash D(0,1 / 2)$ and $z \in \mathbb{D}$ satisfying $\left|z-z_{0}\right| \leq c\left(1-\left|z_{0}\right|\right)$ for a constant $c>0$, there exists $C=C(c, \gamma, \omega)>0$ such that

$$
\int_{\mathbb{D} \backslash D\left(z_{0}, 2\left|z-z_{0}\right|\right)}\left|B_{z_{0}}^{\omega}(\zeta)-B_{z}^{\omega}(\zeta)\right| v(\zeta) d(\omega \otimes m)(\zeta) \leq C \inf _{a \in D\left(z_{0}, \sqrt{2}\left(1-\left|z_{0}\right|\right)\right) \cap \mathbb{D}} M_{\omega}(v)(a) .
$$

Proof. If $\zeta \in \mathbb{D} \backslash D\left(z_{0}, 2\left|z-z_{0}\right|\right)$, then $2\left|z-z_{0}\right| \leq\left|z_{0}-\zeta\right|<\left|1-\bar{\zeta} z_{0}\right|$, and hence

$$
\int_{\mathbb{D} \backslash D\left(z_{0}, 2\left|z-z_{0}\right|\right)}\left|B_{z_{0}}^{\omega}(\zeta)-B_{z}^{\omega}(\zeta)\right| v(\zeta) d(\omega \otimes m)(\zeta) \lesssim\left|z-z_{0}\right| \int_{\mathbb{D}} \frac{\left|B_{z_{0}}^{\omega}(\zeta)\right|}{\left|1-\bar{\zeta} z_{0}\right|} v(\zeta) d(\omega \otimes m)(\zeta)
$$


by Lemma 9, Let $k_{0} \in \mathbb{N}$ such that $2^{k_{0}} \sqrt{2}\left(1-\left|z_{0}\right|\right) \leq 1<2^{k_{0}+1} \sqrt{2}\left(1-\left|z_{0}\right|\right)$. Let $E_{-1}=\emptyset$, $E_{k}=\left\{z \in \mathbb{D}:\left|1-\bar{z}_{0} z\right| \leq 2^{k} \sqrt{2}\left(1-\left|z_{0}\right|\right)\right\}$ for $k=0, \ldots, k_{0}$, and $E_{k_{0}+1}=\mathbb{D} \backslash E_{k_{0}}$. Then, by Lemma 13,

$$
\begin{aligned}
& \left(1-\left|z_{0}\right|\right) \int_{\mathbb{D}} \frac{\left|B_{z_{0}}^{\omega}(\zeta)\right|}{\left|1-\bar{\zeta} z_{0}\right|} v(\zeta) d(\omega \otimes m)(\zeta) \\
& \lesssim\left(1-\left|z_{0}\right|\right) \sum_{k=0}^{k_{0}+1} \int_{E_{k} \backslash E_{k-1}} \frac{1}{\left|1-\bar{\zeta} z_{0}\right|^{\gamma+1}}\left(\int_{0}^{1} \frac{d \nu(r)}{1-r\left(1-\left|1-\bar{\zeta} z_{0}\right|\right)}\right) v(\zeta) d(\omega \otimes m)(\zeta) .
\end{aligned}
$$

Further, Lemma 10 and the hypothesis $\omega \in \widehat{\mathcal{D}}$ give

$$
\begin{aligned}
& \left(1-\left|z_{0}\right|\right) \sum_{k=0}^{k_{0}} \int_{E_{k} \backslash E_{k-1}} \frac{1}{\left|1-\bar{\zeta} z_{0}\right|^{\gamma+1}}\left(\int_{0}^{1} \frac{d \nu(r)}{1-r\left(1-\left|1-\bar{\zeta} z_{0}\right|\right)}\right) v(\zeta) d(\omega \otimes m)(\zeta) \\
& \asymp\left(1-\left|z_{0}\right|\right) \sum_{k=0}^{k_{0}} \int_{E_{k} \backslash E_{k-1}} \frac{1}{\left|1-\bar{\zeta} z_{0}\right|^{2} \widehat{\omega}\left(1-\left|1-\bar{\zeta} z_{0}\right|\right)} v(\zeta) d(\omega \otimes m)(\zeta) \\
& \lesssim \sum_{k=0}^{k_{0}} \frac{1}{2^{2 k}\left(1-\left|z_{0}\right|\right) \widehat{\omega}\left(1-2^{k-1} \sqrt{2}\left(1-\left|z_{0}\right|\right)\right)}(\omega v)\left(E_{k} \backslash E_{k-1}\right) \\
& \leq \sum_{k=0}^{k_{0}} \frac{1}{2^{2 k}\left(1-\left|z_{0}\right|\right) \widehat{\omega}\left(1-2^{k-1} \sqrt{2}\left(1-\left|z_{0}\right|\right)\right)}(\omega v)\left(D\left(z_{0}, 2^{k} \sqrt{2}\left(1-\left|z_{0}\right|\right)\right) \cap \mathbb{D}\right) \\
& \lesssim \sum_{k=0}^{k_{0}} \frac{1}{2^{k} \omega\left(D\left(z_{0}, 2^{k} \sqrt{2}\left(1-\left|z_{0}\right|\right)\right) \cap \mathbb{D}\right)}(\omega v)\left(D\left(z_{0}, 2^{k} \sqrt{2}\left(1-\left|z_{0}\right|\right)\right) \cap \mathbb{D}\right) \\
& \leq M_{a \in D\left(z_{0}, \sqrt{2}\left(1-\left|z_{0}\right|\right)\right) \cap \mathbb{D}} M_{\omega}(v)(a) \sum_{k=0}^{\infty} \frac{1}{2^{k}} \asymp \underset{a \in D\left(z_{0}, \sqrt{2}\left(1-\left|z_{0}\right|\right)\right) \cap \mathbb{D}}{\inf } M_{\omega}(v)(a) .
\end{aligned}
$$

Furthermore, clearly

$$
\begin{aligned}
& \left(1-\left|z_{0}\right|\right) \int_{E_{k_{0}+1}} \frac{1}{\left|1-\bar{\zeta} z_{0}\right|^{\gamma+1}}\left(\int_{0}^{1} \frac{d \nu(r)}{1-r\left(1-\left|1-\bar{\zeta} z_{0}\right|\right)}\right) v(\zeta) d(\omega \otimes m)(\zeta) \\
& \inf _{a \in D\left(z_{0}, \sqrt{2}\left(1-\left|z_{0}\right|\right)\right) \cap \mathbb{D}} M_{\omega}(v)(a),
\end{aligned}
$$

which together with the previous estimate finishes the proof.

Write $\mathbb{D}=\overline{D\left(0, \frac{1}{2}\right)} \cup R_{1} \cup R_{2}$, where $R_{1}$ and $R_{2}$ are dyadic Carleson squares.

Lemma 15. Let $\omega \in \mathcal{R}$ such that $\omega([a, b]) \asymp \omega\left(\left[a, \frac{a+b}{2}\right]\right) \asymp \omega\left(\left[\frac{a+b}{2}, b\right]\right)$ for all $0 \leq a, b \leq 1$, $f \in L_{\omega}^{1}$ and $\lambda>\|f\|_{L_{\omega}^{1}}$. Let $R \in\left\{R_{1}, R_{2}\right\}$. Then there exist $F$ and $\Omega$ such that $R=F \cup \Omega$, $F \cap \Omega=\emptyset$ and

(i) $|f(z)| \leq \lambda$ almost everywhere on $F$;

(ii) $\Omega=\cup_{k} Q_{k}$, where $Q_{k} \subset R$ are dyadic polar rectangles;

(iii) $\omega(\Omega) \leq \frac{\left\|f 1_{R}\right\|_{L_{\omega}^{1}}}{\lambda}$;

(iv) There is a constant $C=C(\omega)>0$ such that $\lambda \leq \frac{1}{\omega\left(Q_{k}\right)} \int_{Q_{k}}|f(z)| d(\omega \otimes m)(z) \leq C \lambda$.

The Calderón-Zygmund decomposition of $f 1_{R}: R \rightarrow \mathbb{C}$ is $f 1_{R}=g+b$, where

$$
g(z)=\left\{\begin{array}{ll}
f(z), & z \in F \\
\frac{1}{\omega\left(Q_{k}\right)} \int_{Q_{k}} f(\zeta) d(\omega \otimes m)(\zeta), & z \in Q_{k}
\end{array} .\right.
$$


Proof. Write $R=Q_{1,0}$ and pick $Q_{1,0}$ if

$$
\frac{1}{\omega\left(Q_{1,0}\right)} \int_{Q_{1,0}}|f(\zeta)| d(\omega \otimes m)(\zeta) \geq \lambda
$$

If not, divide $Q_{1,0}$ into $Q_{k, 0}, j=1, \ldots, 4$, and pick those for which

$$
\frac{1}{\omega\left(Q_{k, 1}\right)} \int_{Q_{k, 1}}|f(\zeta)| d(\omega \otimes m)(\zeta) \geq \lambda
$$

Divide the non-selected ones and proceed. By re-naming the selected sets as $Q_{k}$ and defining $\Omega=\cup_{k} Q_{k}$ we have (ii).

(i) Let $F=R \backslash \Omega$. For almost every $z \in F$ and each $k \in \mathbb{N} \cup\{0\}$ there exists a unique dyadic polar rectangle $Q_{j}$ of generation $j$ such that $z \in Q_{j}$ and

$$
\frac{1}{\omega\left(Q_{j}\right)} \int_{Q_{j}}|f(\zeta)| d(\omega \otimes m)(\zeta) \leq \lambda
$$

Then $\cap_{j} \overline{Q_{j}}=\{z\}$, and hence

$$
|f(z)|=\lim _{j \rightarrow \infty} \frac{1}{\omega\left(Q_{j}\right)} \int_{Q_{j}}|f(\zeta)| d(\omega \otimes m)(\zeta)
$$

for almost every $z \in F$ by Lebesgue's differentiation theorem. It follows that $|f| \leq \lambda$ almost everywhere on $F$.

(iii) Since $\omega\left(Q_{k}\right) \leq \frac{1}{\lambda} \int_{Q_{k}}|f(\zeta)| d(\omega \otimes m)(\zeta)$ for each $k$, we have

$$
\omega(\Omega) \leq \sum_{k} \omega\left(Q_{k}\right) \leq \frac{1}{\lambda} \sum_{k} \int_{Q_{k}}|f(\zeta)| d(\omega \otimes m)(\zeta)=\frac{1}{\lambda} \int_{\Omega}|f(\zeta)| d(\omega \otimes m)(\zeta) \leq \frac{\|f\|_{L_{\omega}^{1}}}{\lambda} .
$$

(iv) Since $\omega(R)=c \omega(\mathbb{D})$ for some constant $c>0$, we have

$$
\frac{1}{\omega(R)} \int_{R}|f(\zeta)| d(\omega \otimes m)(\zeta)=\frac{\left\|f 1_{R}\right\|}{c \omega(\mathbb{D})}<\frac{\lambda}{c \omega(\mathbb{D})} .
$$

For each $Q_{k} \neq R$, there exists a non-selected dyadic polar rectangle $Q^{\prime}$ from the preceding generation such that $Q_{k} \subset Q^{\prime}$. Since $\omega \in \mathcal{R}$ such that $\omega([a, b]) \asymp \omega\left(\left[a, \frac{a+b}{2}\right]\right) \asymp \omega\left(\left[\frac{a+b}{2}, b\right]\right)$ for all $0 \leq a, b \leq 1$ by the hypothesis, we deduce

$$
\lambda>\frac{1}{\omega\left(Q^{\prime}\right)} \int_{Q^{\prime}}|f(\zeta)| d(\omega \otimes m)(\zeta) \geq \frac{1}{C \omega\left(Q_{k}\right)} \int_{Q_{k}}|f(\zeta)| d(\omega \otimes m)(\zeta)
$$

for some constant $C=C(\omega)>0$, and thus (iv) holds.

Proof of Theorem 3. Assume first that $v$ satisfies the $B_{1, \omega}$-condition. Write $\mathbb{D}=\overline{D\left(0, \frac{1}{2}\right)} \cup R_{1} \cup$ $R_{2}$ as before. Then $f=f 1 \frac{}{D\left(0, \frac{1}{2}\right)}+f 1_{R_{1}}+f 1_{R_{2}}$. Since $B_{z}^{\omega}$ is uniformly bounded on $D\left(0, \frac{1}{2}\right)$ and

we have

$$
\operatorname{essinf}_{z \in D\left(0, \frac{1}{2}\right)} v(z) \geq \frac{1}{B_{1, \omega}(v)} \frac{\int_{D\left(0, \frac{1}{2}\right)} v(\zeta) d(\omega \otimes m)(\zeta)}{\omega\left(D\left(0, \frac{1}{2}\right)\right)}
$$

$$
\begin{aligned}
(v \omega)\left(\left\{z:\left|P_{\omega}^{+}\left(f 1 \frac{1}{D\left(0, \frac{1}{2}\right)}\right)(z)\right|>\lambda\right\}\right) & \leq \int_{\mathbb{D}} \frac{\left|P_{\omega}^{+}\left(f 1 \frac{1}{D\left(0, \frac{1}{2}\right)}\right)(z)\right|}{\lambda} v(z) d(\omega \otimes m)(z) \\
& \leq \frac{1}{\lambda} \int_{\mathbb{D}}\left(\int_{D\left(0, \frac{1}{2}\right)}|f(\zeta)|\left|B_{z}^{\omega}(\zeta)\right| d(\omega \otimes m)(\zeta)\right) v(z) d(\omega \otimes m)(z) \\
& \lesssim \frac{1}{\lambda} \int_{D\left(0, \frac{1}{2}\right)}|f(\zeta)| d(\omega \otimes m)(\zeta) \lesssim \frac{B_{1, \omega}(v)\|f\|_{L_{\omega}^{1}(v)}}{\lambda} .
\end{aligned}
$$


Moreover,

$$
\begin{aligned}
(v \omega)\left(\left\{z:\left|P_{\omega}^{+}(f)(z)\right|>\lambda\right\}\right) \leq & (v \omega)\left(\left\{z:\left|P_{\omega}^{+}\left(f 1 \frac{\overline{D\left(0, \frac{1}{2}\right)}}{}\right)(z)\right|+\left|P_{\omega}^{+}\left(f 1_{R_{1}}\right)(z)\right|+\left|P_{\omega}^{+}\left(f 1_{R_{2}}\right)(z)\right|>\lambda\right\}\right) \\
\leq & (v \omega)\left(\left\{z:\left|P_{\omega}^{+}\left(f 1 \frac{\lambda}{D\left(0, \frac{1}{2}\right)}\right)(z)\right|>\frac{\lambda}{3}\right\} \cup\left\{z:\left|P_{\omega}^{+}\left(f 1_{R_{1}}\right)(z)\right|>\frac{\lambda}{3}\right\}\right. \\
& \left.\cup\left\{z:\left|P_{\omega}^{+}\left(f 1_{R_{1}}\right)(z)\right|>\frac{\lambda}{3}\right\}\right) \\
\leq & (v \omega)\left(\left\{z:\left|P_{\omega}^{+}\left(f 1 \overline{D\left(0, \frac{1}{2}\right)}\right)(z)\right|>\frac{\lambda}{3}\right\}\right) \\
& +(v \omega)\left(\left\{z:\left|P_{\omega}^{+}\left(f 1_{R_{1}}\right)(z)\right|>\frac{\lambda}{3}\right\}\right) \\
& +(v \omega)\left(\left\{z:\left|P_{\omega}^{+}\left(f 1_{R_{2}}\right)(z)\right|>\frac{\lambda}{3}\right\}\right)
\end{aligned}
$$

so it suffices to show that

$$
(v \omega)\left(\left\{z:\left|P_{\omega}^{+}\left(f 1_{R}\right)(z)\right|>\lambda\right\}\right) \lesssim \frac{\left\|f 1_{R}\right\|_{L_{\omega}^{1}(v)}}{\lambda}, \quad R \in\left\{R_{1}, R_{2}\right\},
$$

for large values of $\lambda$. To see this, fix $R \in\left\{R_{1}, R_{2}\right\}$, and decompose $\left|f 1_{R}\right|=g+b$ according to Lemma 15 and the weight $\omega \in \mathcal{R}$. Then the definition of $g$ and Lemma 15)(iv) give

$$
|g(z)| \leq \sum_{k}\left(\frac{1_{Q_{k}}(z)}{\omega\left(Q_{k}\right)} \int_{Q_{k}}|f(\zeta)| d(\omega \otimes m)(\zeta)\right) \lesssim \lambda, \quad z \in \Omega=\cup_{k} Q_{k},
$$

which together with Lemma 15(i) and the definition of $g$ yields

$$
\begin{aligned}
\|g\|_{L_{v \omega}^{2}}^{2} & =\int_{F}|g(\zeta)|^{2} v(\zeta) d(\omega \otimes m)(\zeta)+\int_{\Omega}|g(\zeta)|^{2} v(\zeta) d(\omega \otimes m)(\zeta) \\
& \lesssim \lambda \int_{F}|f(\zeta)| v(\zeta) d(\omega \otimes m)(\zeta)+\lambda \int_{\Omega}|g(\zeta)| v(\zeta) d(\omega \otimes m)(\zeta) .
\end{aligned}
$$

Now, since $v \in B_{1, \omega}$ and $\omega \in \mathcal{R}$ such that $\omega([a, b]) \asymp \omega\left(\left[a, \frac{a+b}{2}\right]\right) \asymp \omega\left(\left[\frac{a+b}{2}, b\right]\right)$ for all $0 \leq a, b \leq 1$ by the hypotheses,

$$
\begin{aligned}
\int_{\Omega}|g(\zeta)| v(\zeta) d(\omega \otimes m)(\zeta) & =\sum_{k} \int_{Q_{k}}|f(\zeta)| \frac{(v \omega)\left(Q_{k}\right)}{\omega\left(Q_{k}\right)} d(\omega \otimes m)(\zeta) \\
& \lesssim \sum_{k} \int_{Q_{k}}|f(\zeta)| M_{\omega}(v)(\zeta) d(\omega \otimes m)(\zeta) \\
& \leq B_{1, \omega}(v) \sum_{k} \int_{Q_{k}}|f(\zeta)| v(\zeta) d(\omega \otimes m)(\zeta) \\
& =B_{1, \omega}(v) \int_{\Omega}|f(\zeta)| v(\zeta) d(\omega \otimes m)(\zeta) .
\end{aligned}
$$

Therefore $\|g\|_{L_{\omega}^{2}(v)}^{2} \lesssim \lambda B_{1, \omega}(v)\left\|f 1_{R}\right\|_{L_{\omega}^{1}(v)}$, and thus $g \in L_{\omega}^{2}(v)$. Since $B_{1, \omega} \subset B_{2, \omega}$ with $B_{2, \omega}(v) \lesssim B_{1, \omega}(v), P_{\omega}^{+}: L_{\omega}^{2}(v) \rightarrow L_{\omega}^{2}(v)$ is bounded by Theorem 2. Consequently,

$$
\begin{aligned}
(v \omega)\left(\left\{z:\left|P_{\omega}^{+}(g)(z)\right|>\lambda\right\}\right) & =(v \omega)\left(\left\{z:\left|P_{\omega}^{+}(g)(z)\right|^{2}>\lambda^{2}\right\}\right) \\
& \leq \frac{1}{\lambda^{2}} \int_{\mathbb{D}}\left|P_{\omega}^{+}(g)(\zeta)\right|^{2} v(\zeta) d(\omega \otimes m)(\zeta) \lesssim B_{2, \omega}(v) \frac{\|g\|_{L_{\omega}^{2}(v)}^{2}}{\lambda^{2}} \\
& \lesssim B_{1, \omega}^{2}(v) \frac{\left\|f 1_{R}\right\|_{L_{\omega}^{1}(v)}}{\lambda} .
\end{aligned}
$$

To deal with $b$, write $b=\sum_{k} b_{k}$, where $b_{k}=b 1_{Q_{k}}$. Then $\left|P_{\omega}^{+}(b)\right| \leq \sum_{k}\left|P_{\omega}^{+}\left(b_{k}\right)\right|$. For each $k$, let $D_{k}$ be the circumscribed disc of $Q_{k}$ with center $z_{k}$ and let $D_{k}^{\prime}$ be the concentric 
disc of double radius. Further, let $\Omega^{\prime}=\cup_{k} D_{k}^{\prime} \cap \mathbb{D}$. Now that $b$ has mean value zero on $Q_{k}$,

$$
\begin{aligned}
\int_{Q_{k}} b(\zeta) d(\omega \otimes m)(\zeta)= & \int_{Q_{k}}(f(\zeta)-g(\zeta)) d(\omega \otimes m)(\zeta)=\int_{Q_{k}} f(\zeta) d(\omega \otimes m)(\zeta) \\
& -\int_{Q_{k}}\left(\frac{1}{\omega\left(Q_{k}\right)} \int_{Q_{k}} f(z) d(\omega \otimes m)(z)\right) d(\omega \otimes m)(\zeta)=0,
\end{aligned}
$$

we deduce

$$
\begin{aligned}
\left|P_{\omega}^{+}\left(b_{k}\right)(z)\right| & =\left|\int_{Q_{k}} b(\zeta)\right| \overline{B_{z}^{\omega}(\zeta)}\left|d(\omega \otimes m)(\zeta)-\int_{Q_{k}} b(\zeta)\right| \overline{B_{z}^{\omega}\left(z_{k}\right)}|d(\omega \otimes m)(\zeta)| \\
& \leq \int_{Q_{k}}|b(\zeta)|\left|B_{z}^{\omega}(\zeta)-B_{z}^{\omega}\left(z_{k}\right)\right| d(\omega \otimes m)(\zeta) .
\end{aligned}
$$

Consequently,

$$
\begin{aligned}
& \int_{\mathbb{D} \backslash \Omega^{\prime}}\left|P_{\omega}^{+}(b)(z)\right| v(z) d(\omega \otimes m)(z) \\
& \leq \sum_{k} \int_{\mathbb{D} \backslash \Omega^{\prime}}\left|P_{\omega}^{+}\left(b_{k}\right)(z)\right| v(z) d(\omega \otimes m)(z) \\
& \leq \sum_{k} \int_{\mathbb{D} \backslash \Omega^{\prime}}\left(\int_{Q_{k}}|b(\zeta)|\left|B_{z}^{\omega}(\zeta)-B_{z}^{\omega}\left(z_{k}\right)\right| d(\omega \otimes m)(\zeta)\right) v(z) d(\omega \otimes m)(z) \\
& =\sum_{k} \int_{Q_{k}}|b(\zeta)|\left(\int_{\mathbb{D} \backslash \Omega^{\prime}}\left|B_{z}^{\omega}(\zeta)-B_{z}^{\omega}\left(z_{k}\right)\right| v(z) d(\omega \otimes m)(z)\right) d(\omega \otimes m)(\zeta) \\
& \leq \sum_{k} \int_{Q_{k}}|b(\zeta)|\left(\int_{\mathbb{D} \backslash D\left(z_{k}, 2\left|\zeta-z_{k}\right|\right)}\left|B_{z}^{\omega}(\zeta)-B_{z}^{\omega}\left(z_{k}\right)\right| v(z) d(\omega \otimes m)(z)\right) d(\omega \otimes m)(\zeta) .
\end{aligned}
$$

There is an absolute constant $C>0$ such that $\left|\zeta-z_{k}\right| \leq C\left(1-\left|z_{k}\right|\right)$ for any $k$ and any $\zeta \in Q_{k}$. Hence the inner integral in each summand is bounded by a constant times $\inf _{a \in D\left(z_{k}, \sqrt{2}\left(1-\left|z_{k}\right|\right)\right.} M_{\omega}(v)(a)$ by Lemma 14. Therefore (4.3) yields

$$
\begin{aligned}
\int_{\mathbb{D} \backslash \Omega^{\prime}}\left|P_{\omega}^{+}(b)(z)\right| v(z) d(\omega \otimes m)(z) & \lesssim \sum_{k} \inf _{a \in D\left(z_{k}, \sqrt{2}\left(1-\left|z_{k}\right|\right)\right.} M_{\omega}(v)(a) \int_{Q_{k}}|b(\zeta)| d(\omega \otimes m)(\zeta) \\
\leq & \sum_{k} \int_{Q_{k}}|b(\zeta)| M_{\omega}(v)(\zeta) d(\omega \otimes m)(\zeta) \\
\leq & B_{1, \omega}(v) \sum_{k} \int_{Q_{k}}|b(\zeta)| v(\zeta) d(\omega \otimes m)(\zeta) \\
\leq & B_{1, \omega}(v) \int_{\Omega}|b(\zeta)| v(\zeta) d(\omega \otimes m)(\zeta) \\
\leq & B_{1, \omega}(v) \int_{\Omega}|f(\zeta)| v(\zeta) d(\omega \otimes m)(\zeta) \\
& +B_{1, \omega}(v) \int_{\Omega}|g(\zeta)| v(\zeta) d(\omega \otimes m)(\zeta) \\
& B_{1, \omega}^{2}(v) \int_{\Omega}|f(\zeta)| v(\zeta) d(\omega \otimes m)(\zeta) \\
\lesssim & B_{1, \omega}^{2}(v)\left\|f 1_{R}\right\|_{L_{\omega}^{1}(v)} .
\end{aligned}
$$


Further,

$$
\begin{aligned}
(v \omega)\left(\left\{z:\left|P_{\omega}^{+}(b)(z)\right|>\lambda\right\}\right) \leq & (v \omega)\left(\left\{z:\left|P_{\omega}^{+}(b)(z)\right|>\lambda\right\} \cap\left(\mathbb{D} \backslash \Omega^{\prime}\right)\right) \\
& +(v \omega)\left(\left\{z:\left|P_{\omega}^{+}(b)(z)\right|>\lambda\right\} \cap \Omega^{\prime}\right),
\end{aligned}
$$

where

$$
(v \omega)\left(\left\{z:\left|P_{\omega}^{+}(b)(z)\right|>\lambda\right\} \cap\left(\mathbb{D} \backslash \Omega^{\prime}\right)\right) \leq \frac{1}{\lambda} \int_{\mathbb{D} \backslash \Omega^{\prime}}\left|P_{\omega}^{+}(b)(z)\right| v(z) d(\omega \otimes m)(z) \lesssim \frac{\left\|f 1_{R}\right\|_{L_{\omega}^{1}(v)}}{\lambda}
$$

by (4.5). Since $\omega \in \mathcal{R}$ such that $\omega([a, b]) \asymp \omega\left(\left[a, \frac{a+b}{2}\right]\right) \asymp \omega\left(\left[\frac{a+b}{2}, b\right]\right)$ for all $0 \leq a, b \leq 1$ by the hypothesis, we have $\omega\left(Q_{k}\right) \asymp \omega\left(D_{k}^{\prime} \cap \mathbb{D}\right)$, and hence (iv) gives

$$
\begin{aligned}
& (v \omega)\left(\left\{z:\left|P_{\omega}^{+}(b)(z)\right|>\lambda\right\} \cap \Omega^{\prime}\right) \leq(v \omega)\left(\Omega^{\prime}\right) \leq \sum_{k}(v \omega)\left(D_{k}^{\prime} \cap \mathbb{D}\right) \\
& \leq \frac{1}{\lambda} \sum_{k} \frac{\left.(v \omega)\left(D_{k}^{\prime} \cap \mathbb{D}\right)\right)}{\omega\left(Q_{k}\right)} \int_{Q_{k}}|f(z)| d(\omega \otimes m)(z) \\
& \lesssim \frac{1}{\lambda} \sum_{k} \frac{\left.(v \omega)\left(D_{k}^{\prime} \cap \mathbb{D}\right)\right)}{\omega\left(D_{k}^{\prime} \cap \mathbb{D}\right)} \int_{Q_{k}}|f(z)| d(\omega \otimes m)(z) \\
& \leq \frac{1}{\lambda} \sum_{k} \int_{Q_{k}}|f(z)| M_{\omega}(v)(z) d(\omega \otimes m)(z) \\
& \leq B_{1, \omega}(v) \frac{1}{\lambda} \sum_{k} \int_{Q_{k}}|f(z)| v(z) d(\omega \otimes m)(z) \leq B_{1, \omega}(v) \frac{\left\|f 1_{R}\right\|_{L_{\omega}^{1}(v)}}{\lambda} .
\end{aligned}
$$

Hence

$$
\begin{aligned}
(v \omega)\left(\left\{z:\left|P_{\omega}^{+}\left(f 1_{R}\right)(z)\right|>\lambda\right\}\right) \leq & (v \omega)\left(\left\{z:\left|P_{\omega}^{+}(g)(z)\right|>\lambda / 2\right\}\right) \\
& +(v \omega)\left(\left\{z:\left|P_{\omega}^{+}(b)(z)\right|>\lambda / 2\right\}\right) \\
\lesssim & \frac{\left\|f 1_{R}\right\|_{L_{\omega}^{1}(v)}}{\lambda}, \quad R \in\left\{R_{1}, R_{2}\right\},
\end{aligned}
$$

and thus we get (i). To be precise, this proof works only for $f \in L_{\omega}^{1}$ because Lemma 15 is applied, but the general case follows by applying (4.7) to the function $\min \{f, n\}$ with $f$ non-negative and then letting $n \rightarrow \infty$.

Since (i) trivially implies (ii), it remains to show that (ii) implies (iii). Let $S_{1}$ and $S_{2}$ be Carleson squares satisfying the hypothesis in Lemma 11, and let $f$ a non-negative function supported on $S_{1}$. Further, choose

$$
\lambda=C \frac{\int_{S_{1}} f(\zeta) d(\omega \otimes m)(\zeta)}{\omega\left(S_{1}\right)}
$$

where $C$ is the constant appearing in (3.14). Since $\lambda(v \omega)\left(\left\{z:\left|P_{\omega}(f)(z)\right|>\lambda\right\}\right) \lesssim\|f\|_{L_{\omega}^{1}(v)}$ by the hypothesis, it follows by Lemma 11 that there exists $C_{1}>0$ such that

$$
\frac{(f \omega)\left(S_{1}\right)}{\omega\left(S_{1}\right)}(v \omega)\left(S_{2}\right) \leq C_{1}(f v \omega)\left(S_{1}\right) .
$$

By choosing $f=1_{E} \omega^{-1}$ for $E \subset S_{1}$ and applying Lebesgue differentiation theorem, we get

$$
\frac{(v \omega)\left(S_{2}\right)}{\omega\left(S_{1}\right)} \leq C_{1} v(z)
$$

for almost every $z \in S_{1}$. Since the same is true when the roles of $S_{1}$ and $S_{2}$ are interchanged, we deduce

$$
C_{1} v(z) \geq \frac{(v \omega)\left(S_{2}\right)}{\omega\left(S_{1}\right)} \geq \frac{\omega\left(S_{2}\right)(v \omega)\left(S_{1}\right)}{C_{1} \omega\left(S_{2}\right) \omega\left(S_{1}\right)}=\frac{1}{C_{1}} \frac{(v \omega)\left(S_{1}\right)}{\omega\left(S_{1}\right)}
$$


for almost every $z \in S_{1}$. It follows that

$$
\sup _{S: z \in S} \frac{(v \omega)(S)}{\omega(S)} \lesssim v(z)
$$

for almost every $z \in \mathbb{D}$. This implies

$$
\sup _{z \in D(a, r)} \frac{(v \omega)(D(a, r) \cap \mathbb{D})}{\omega(D(a, r) \cap \mathbb{D})} \lesssim v(z)
$$

for almost every $z \in \mathbb{D}$, where the supremum runs over the discs touching the boundary. Moreover, the squares $S_{1}$ and $S_{2}$ in the statement of Lemma 11 can be replaced by Euclidean discs $D\left(a_{1}, R\left(1-\left|a_{1}\right|\right)\right.$ and $D\left(a_{2}, R\left(1-\left|a_{1}\right|\right)\right.$, where $R$ is fixed and small enough. By using this fact with the above reasoning in hand and (4.8), we deduce $v \in B_{1, \omega}$.

\section{Two-Weight Inequality FOR The POSITIVE OPERATOR $P_{\Psi, \mu}^{+}$}

The purpose of this section is to prove Theorem 16, A reasoning similar to that in the proof of Theorem 2 then shows that Theorem 4 is an immediate consequence of this result.

Theorem 16. Let $1<p<\infty$ and $\mu$ be a positive Borel measure on $\mathbb{D}$, and let $v, u \in L_{\mu}^{1}$ non-negative. Let $\Psi: D(1,1) \rightarrow \mathbb{C}$ be an analytic function such that its restriction to the interval $(0,2)$ is positive and the following conditions hold:

(i) $|\Psi(1-z)| \asymp \Psi(|1-z|)$ for all $z \in \mathbb{D}$;

(ii) $\Psi$ is essentially decreasing on $(0,2)$;

(iii) There exists a constant $C>0$ such that $\Psi(t) \leq C \Psi(2 t)$ for all $t \in(0,1)$;

(iv) $|\Psi(1-z)|=|\Psi(1-\bar{z})|$ for all $z \in \mathbb{D}$.

Then $P_{\Psi, \mu}^{+}: L_{\mu}^{p}(v) \rightarrow L_{\mu}^{p}(u)$ is bounded if and only if there exist constants $C_{0}=C_{0}(p, \mu, v, u)>$ 0 and $C_{0}^{\star}=C_{0}^{\star}(p, \mu, v, u)>0$ such that

$$
\left\|\mathcal{M}_{u^{1 / p}} P_{\Psi, \mu}^{+} \mathcal{M}_{\sigma^{1 / p^{\prime}}}\left(1_{S} \sigma^{1 / p}\right)\right\|_{L_{\mu}^{p}} \leq C_{0}\left\|1_{S} \sigma^{1 / p}\right\|_{L_{\mu}^{p}}
$$

and

$$
\left\|\mathcal{M}_{\sigma^{1 / p^{\prime}}} P_{\Psi, \mu}^{+} \mathcal{M}_{u^{1 / p}}\left(1_{S} u^{1 / p^{\prime}}\right)\right\|_{L_{\mu}^{p^{\prime}}} \leq C_{0}^{\star}\left\|1_{S} u^{1 / p^{\prime}}\right\|_{L_{\mu}^{p^{\prime}}}
$$

for all Carleson squares $S \subset \mathbb{D}$, where $\sigma=v^{1-p^{\prime}}$. Moreover, there exists a constant $C_{1}=$ $C_{1}(p, \mu)>0$ such that

$$
\left\|P_{\Psi, \mu}^{+}\right\|_{L_{\mu}^{p}(v) \rightarrow L_{\mu}^{p}(u)} \leq C_{1}\left(C_{0}+C_{0}^{\star}\right) .
$$

As in the one-weight case $P_{\Psi, \mu}^{+}: L_{\mu}^{p}(v) \rightarrow L_{\mu}^{p}(v)$ given in Corollary 8 it is more convenient to consider first a dyadic model. To do this, let $\mathbb{E}_{S}^{\mu} f$ and $\mathbb{E}_{S}^{\sigma \mu} f$ denote the expectations of a function $f$ over a square $S$ with respect to the measures $\mu$ and $\sigma d \mu$, respectively. Given a dyadic grid $\mathcal{D}$ on $\mathbb{T}$ and a sequence $\tau=\left\{\tau_{S(I)}\right\}_{I \in \mathcal{D}}$ of nonnegative numbers, consider the dyadic positive operator defined by

$$
T(f)=T_{\mu, \tau, \mathcal{D}}(f)=\sum_{I \in \mathcal{D}} \tau_{S(I)}\left(\mathbb{E}_{S(I)}^{\mu} f\right) 1_{S(I)} .
$$

Given $I \subset \mathcal{D}$ we can identify it with its associated Carleson square $S(I)$. So, via this identification, for a dyadic grid $\mathcal{D}$ on $\mathbb{T}$ we shall simply write

$$
T(f)=T_{\mu, \tau, \mathcal{D}}(f)=\sum_{I \in \mathcal{D}} \tau_{S}\left(\mathbb{E}_{S}^{\mu} f\right) 1_{S}
$$

for the corresponding dyadic positive operator.

The following theorem characterizes the boundedness of the operator $T$ in the two-weight setting. See [15, 17, 21]. 
Theorem 17. Let $1<p<\infty, \mu$ be a positive Borel measure on $\mathbb{D}, \sigma, u \in L_{\mu}^{1}$ non-negative and let $T=T_{\mu, \tau, \mathcal{D}}$ be the dyadic positive operator defined in (5.4). Then $T(\sigma \cdot): L_{\mu}^{p}(\sigma) \rightarrow L_{\mu}^{p}(u)$ is bounded if and only if there exist constants $C_{0}=C_{0}(p, \mu, \sigma, u)>0$ and $C_{0}^{\star}=C_{0}^{\star}(p, \mu, \sigma, u)>0$ such that

and

$$
\left\|T\left(\sigma 1_{S}\right)\right\|_{L_{\mu}^{p}(u)}^{p} \leq C_{0}(\sigma \mu)(S)
$$

$$
\left\|T^{\star}\left(u 1_{S}\right)\right\|_{L_{\mu}^{p^{\prime}(\sigma)}}^{p^{\prime}} \leq C_{0}^{\star}(u \mu)(S)
$$

for all $S \in \mathcal{D}$. Moreover, there exists a constant $C_{1}=C_{1}(p, \mu)>0$ such that

$$
\|T(\sigma \cdot)\|_{L_{\mu}^{p}(\sigma) \rightarrow L_{\mu}^{p}(u)} \leq C_{1}\left(C_{0}+C_{0}^{\star}\right) .
$$

Let now $\sigma$ be a weight and $f$ a locally integrable function in $\mathbb{D}$. Let $S_{0} \in \mathcal{D}$ and denote $\mathcal{D}_{0}=\left\{S \in \mathcal{D}: S \subset S_{0}\right\}$. Further, let

$\mathcal{L}\left(S_{0}\right)=\left\{S \in \mathcal{D}_{0}: S\right.$ is a maximal Carleson square in $\mathcal{D}_{0}$ such that $\left.\mathbb{E}_{S}^{\sigma \mu}|f|>4 \mathbb{E}_{S_{0}}^{\sigma \mu}|f|\right\}$.

Define $\mathcal{L}_{0}=\left\{S_{0}\right\}$ and $\mathcal{L}_{i}=\cup_{L \in \mathcal{L}_{i-1}} \mathcal{L}(L)$ for all $i \in \mathbb{N}$, and denote the union of all the stopping squares by $\mathcal{L}=\cup_{i \geq 0} \mathcal{L}_{i}$. For $S \in \mathcal{D}_{0}$, let $\lambda(S)$ be the minimal square $L \in \mathcal{L}$ such that $S \subset L$ and let $\mathcal{D}(L)=\left\{S \in \mathcal{D}_{0}: \lambda(S)=L\right\}$.

The stopping squares $\mathcal{L}$ can be used to linearise the maximal function $M_{\nu}$. More precisely, we have the pointwise estimate

$$
\sum_{L \in \mathcal{L}}\left(\mathbb{E}_{L}^{\sigma \mu}|f|\right) 1_{L}(z) \lesssim M_{\sigma \mu} f(z), \quad z \in \mathbb{D} .
$$

To see this, assume $z \in S_{0}$ for some $S_{0} \in \mathcal{L}_{0}$, for otherwise the inequality is trivial because the left hand side is zero. Then there exists a stopping square $L^{\prime} \in \mathcal{L}$ with minimal side length containing $z$. The expectations increase geometrically, that is,

therefore

$$
\mathbb{E}_{L}^{\sigma \mu}|f|>4 \mathbb{E}_{\widetilde{L}}^{\sigma \mu}|f|, \quad L, \widetilde{L} \in \mathcal{L}, \quad L \subsetneq \widetilde{L}
$$

$$
\sum_{\substack{L \in \mathcal{L} \\ z \in L}} \mathbb{E}_{L}^{\sigma \mu}|f| \leq \mathbb{E}_{L^{\prime}}^{\sigma \mu}|f| \sum_{j=0}^{\infty} 4^{-j} \lesssim M_{\sigma \mu} f(z),
$$

concluding the proof of (5.7).

An application of (5.7) and (3.9) provides the useful inequality

$$
\sum_{L \in \mathcal{L}}\left(\mathbb{E}_{L}^{\sigma \mu}|f|\right)^{p} \sigma \mu(L) \lesssim\|f\|_{L_{\mu}^{p}(\sigma)} .
$$

Proof of Theorem 17. We will assume there is a finite collection of dyadic squares $\mathcal{S}$ in the definition of the operator $T$, and we will prove the operator norm is independent of the chosen collection. So from now on

$$
T f=\sum_{S \in \mathcal{S}} \tau_{S}\left(\mathbb{E}_{S}^{\mu} f\right) 1_{S}
$$

It is enough to prove boundedness of the bilinear form $\langle T(\sigma f), g u\rangle_{L_{\mu}^{2}}$, where $f \in L_{\mu}^{p}(\sigma)$ and $g \in L_{\mu}^{p^{\prime}}(u)$ are positive. Following the argument in [21], we seek an estimate of the form

$$
\langle T(\sigma f), g u\rangle_{L_{\mu}^{2}} \leq A\|f\|_{L_{\mu}^{p}(\sigma)}\|g\|_{L_{\mu}^{p^{\prime}(u)}}+B\|f\|_{L_{\mu}^{p}(\sigma)}^{p} .
$$

We first divide the squares in $\mathcal{S}$ into two collections $\mathcal{S}_{1}$ and $\mathcal{S}_{2}$ according to the following criterion. A square $S$ will belong to $\mathcal{S}_{1}$, if

$$
\left(\mathbb{E}_{S}^{\mu \sigma} f\right)^{p} \mu \sigma(S) \geq\left(\mathbb{E}_{S}^{\mu u} g\right)^{p^{\prime}} \mu u(S)
$$

and it will belong to $\mathcal{S}_{2}$ otherwise. This reorganization of the Carleson squares allows us to write $T=T_{1}+T_{2}$, where

$$
T_{i} f=\sum_{S \in \mathcal{S}_{i}} \tau_{S}\left(\mathbb{E}_{S}^{\mu} f\right) 1_{S}, \quad i=1,2
$$


The idea of writing $T$ as the sum of $T_{1}$ and $T_{2}$ was already present in the work of Treil [21] and previously in the work of Nazarov, Treil and Volberg [10]. We will prove boundedness of $T_{1}$ using the testing condition (5.5). The boundedness of $T_{2}$ can be proven analogously to $T_{1}$, only using (5.6) this time. First note that

$$
\begin{aligned}
\left\langle T_{1}(\sigma f), g u\right\rangle_{L_{\mu}^{2}} & =\sum_{S \in \mathcal{S}_{1}} \tau_{S} \mathbb{E}_{S}^{\mu}(f \sigma)\left\langle g u, 1_{S}\right\rangle_{L_{\mu}^{2}}=\sum_{L \in \mathcal{L}} \sum_{S \in \mathcal{D}(L)} \tau_{S} \mathbb{E}_{S}^{\mu}(f \sigma)\left\langle g u, 1_{S}\right\rangle_{L_{\mu}^{2}} \\
& =\sum_{L \in \mathcal{L}}\left\langle T_{L}(\sigma f), g u\right\rangle_{L_{\mu}^{2}}
\end{aligned}
$$

where $\mathcal{L}$ is a collection of stopping Carleson squares in the family $\mathcal{S}_{1}$, to be specified below, and $T_{L} f=\sum_{S \in \mathcal{D}(L)} \tau_{S} \mathbb{E}_{S}^{\mu}(f) 1_{S}$. To find the collection of stopping Carleson squares $\mathcal{L}$, we define $\mathcal{L}_{0}$ as the collection of maximal Carleson squares in the family $\mathcal{S}_{1}$, and follow the definition after Theorem 17 for given $f$ and $\sigma$ to define $\mathcal{L}$, with $\mathcal{S}_{1}$ as our family of dyadic Carleson squares. Clearly,

$$
\sum_{L \in \mathcal{L}}\left\langle T_{L}(\sigma f), g u\right\rangle_{L_{\mu}^{2}}=\sum_{L \in \mathcal{L}} \int T_{L}(\sigma f)(z) g(z) u(z) d \mu(z)=I+I I,
$$

where

$$
I=\sum_{i} \sum_{L \in \mathcal{L}_{i}} \int_{L \backslash \cup_{\substack{L^{\prime} \in \mathcal{L}_{i+1} \\ L^{\prime} \subset L}} L^{\prime}} T_{L}(\sigma f)(z) g(z) u(z) d \mu(z)
$$

and

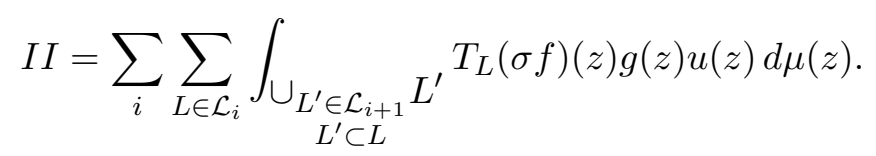

To deal with $I$, we estimate the norm of $T_{L}$. By using the testing condition (5.5) and the fact that $S \in \mathcal{D}(L)$ are not stopping Carleson squares, we deduce

$$
\begin{aligned}
\left\|T_{L}(\sigma f)\right\|_{L_{\mu}^{p}(u)}^{p} & =\left\|\sum_{S \in \mathcal{D}(L)} \tau_{S} \mathbb{E}_{S}^{\mu}(f \sigma) 1_{S}\right\|_{L_{\mu}^{p}(u)}^{p}=\left\|\sum_{S \in \mathcal{D}(L)} \frac{\mu \sigma(S)}{\mu(S)} \tau_{S} \mathbb{E}_{S}^{\sigma \mu}(f) 1_{S}\right\|_{L_{\mu}^{p}(u)}^{p} \\
& \leq 4^{p}\left(\mathbb{E}_{L}^{\sigma \mu}(f)\right)^{p}\left\|\sum_{S \in \mathcal{D}(L)} \frac{\mu \sigma(S)}{\mu(S)} \tau_{S} 1_{S}\right\|_{L_{\mu}^{p}(u)}^{p} \\
& \leq 4^{p}\left(\mathbb{E}_{L}^{\sigma \mu}(f)\right)^{p}\left\|T\left(\sigma 1_{L}\right)\right\|_{L_{\mu}^{p}(u)}^{p} \leq 4^{p} C_{0}\left(\mathbb{E}_{L}^{\sigma \mu}(f)\right)^{p} \sigma \mu(L) .
\end{aligned}
$$

Since $\cup_{i} \cup_{L \in \mathcal{L}_{i}} L \backslash \cup_{\substack{L^{\prime} \in \mathcal{L}_{i+1} \\ L^{\prime} \subset L}} L^{\prime}$ forms a collection of disjoint sets in $\mathcal{L}_{0}$, Hölder's inequality, (5.12) and (5.8) yield

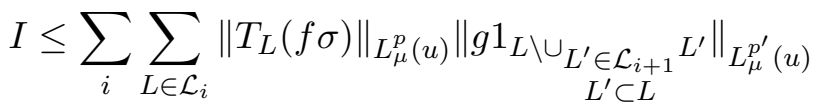

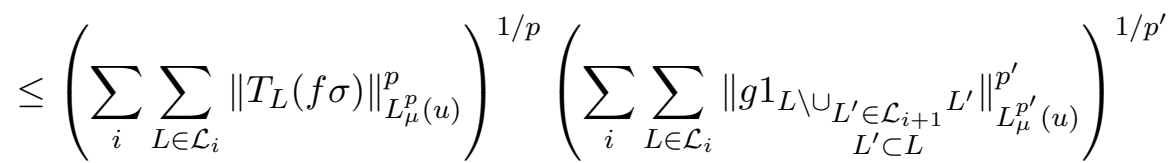

$$
\begin{aligned}
& \leq 4 C_{0}^{1 / p}\left(\sum_{L \in \mathcal{L}}\left(\mathbb{E}_{L}^{\mu \sigma} f\right)^{p} \sigma \mu(L)\right)^{1 / p}\|g\|_{L_{\mu}^{p^{\prime}(u)}} \lesssim C_{0}\|f\|_{L_{\mu}^{p}(\sigma)}\|g\|_{L_{\mu}^{p^{\prime}}(u)} .
\end{aligned}
$$

We now turn to II. If $L \in \mathcal{L}$ be fixed, then the operator $T_{L}(f \sigma)$ is constant on $L^{\prime}$, where $L^{\prime} \in \mathcal{L}, L^{\prime} \subsetneq L$. That is, $L^{\prime}$ is contained in some Carleson square $S$ of the family $\mathcal{D}(L)$. We 
will denote this constant by $T_{L}(f \sigma)\left(L^{\prime}\right)$. For a fixed $L \in \mathcal{L}_{i}$, this, Hölder's inequality, (5.12) and the hypothesis (5.10) yield

$$
\begin{aligned}
& \int_{\substack{\cup_{L^{\prime} \in \mathcal{L}_{i+1}} L^{\prime} \\
L^{\prime} \subset L}} T_{L}(\sigma f)(z) g(z) u(z) d \mu(z)=\sum_{\substack{L^{\prime} \in \mathcal{L}_{i+1} \\
L^{\prime} \subset L}} T_{L}(f \sigma)\left(L^{\prime}\right) \int_{L^{\prime}} g(z) u(z) d \mu(z) \\
& =\sum_{\substack{L^{\prime} \in \mathcal{L}_{i+1} \\
L^{\prime} \subset L}} T_{L}(f \sigma)\left(L^{\prime}\right) \frac{\int_{L^{\prime}} g(z) u(z) d \mu(z)}{\mu u\left(L^{\prime}\right)} \mu u\left(L^{\prime}\right) \\
& =\sum_{\substack{L^{\prime} \in \mathcal{L}_{i+1} \\
L^{\prime} \subset L}} \int_{L^{\prime}} T_{L}(f \sigma)(z)\left(\mathbb{E}_{L^{\prime}}^{\mu u} g\right) u(z) d \mu(z) \\
& =\int_{L} T_{L}(f \sigma)(z)\left(\sum_{\substack{L^{\prime} \in \mathcal{L}_{i+1} \\
L^{\prime} \subset L}} \mathbb{E}_{L^{\prime}}^{\mu u} g 1_{L^{\prime}}(z)\right) u(z) d \mu(z) \\
& \leq\left\|T_{L}(f \sigma)\right\|_{L_{\mu}^{p}(u)}\left\|\sum_{\substack{L^{\prime} \in \mathcal{L}_{i+1} \\
L^{\prime} \subset L}} \mathbb{E}_{L^{\prime}}^{\mu u} g 1_{L^{\prime}}\right\|_{\substack{L_{\mu}^{p^{\prime}}(u)\\
}} \\
& =\left\|T_{L}(f \sigma)\right\|_{L_{\mu}^{p}(u)}\left(\sum_{\substack{L^{\prime} \in \mathcal{L}_{i+1} \\
L^{\prime} \subset L}}\left(\mathbb{E}_{L^{\prime}}^{\mu u} g\right)^{p^{\prime}} \mu u\left(L^{\prime}\right)\right)^{1 / p^{\prime}} \\
& \leq 4 C_{0}\left(\mathbb{E}_{L}^{\mu \sigma} f\right) \mu \sigma(L)^{1 / p}\left(\sum_{\substack{L^{\prime} \in \mathcal{L}_{i+1} \\
L^{\prime} \subset L}}\left(\mathbb{E}_{L^{\prime}}^{\mu \sigma} f\right)^{p} \mu \sigma\left(L^{\prime}\right)\right)^{1 / p^{\prime}} .
\end{aligned}
$$

By summing this estimate in $L$ and using (5.8), we obtain

$$
\begin{aligned}
I I & \lesssim \sum_{i} \sum_{L \in \mathcal{L}_{i}}\left(\mathbb{E}_{L}^{\mu \sigma} f\right) \mu \sigma(L)^{1 / p}\left(\sum_{\substack{L^{\prime} \in \mathcal{L}_{i+1} \\
L^{\prime} \subset L}}\left(\mathbb{E}_{L^{\prime}}^{\mu \sigma} f\right)^{p} \mu \sigma\left(L^{\prime}\right)\right)^{1 / p^{\prime}} \\
& \lesssim\left(\sum_{L \in \mathcal{L}}\left(\mathbb{E}_{L}^{\mu \sigma} f\right)^{p} \mu \sigma(L)\right)^{1 / p}\left(\sum_{i} \sum_{L \in \mathcal{L}_{i}} \sum_{L^{\prime} \in \mathcal{L}_{i+1}}\left(\mathbb{E}_{L^{\prime} \subset L}^{\mu \sigma} f\right)^{p} \mu \sigma\left(L^{\prime}\right)\right)^{1 / p^{\prime}} \\
& \lesssim\|f\|_{L_{\mu}^{p}(\sigma)}\|f\|_{L_{\mu}^{p}(\sigma)}^{p / p^{\prime}} \lesssim\|f\|_{L_{\mu}^{p}(\sigma)}^{p} .
\end{aligned}
$$

By combining (5.13) and (5.14), we get (5.9).

We now turn to the two-weight inequality for the case of the operator $P_{\Psi, \mu}^{+}$and its associated dyadic model $P_{\Psi, \mu}^{\beta}$.

Taking $\mathcal{D}^{\beta}, \beta \in\{0,1 / 2\}$, one of the dyadic grids on $\mathbb{T}$ defined in (3.2) and choosing $\tau_{S(I)}=$ $\frac{\Psi(|I|) \mu\left(S_{I}\right)}{|I|}$, we obtain the following result as a byproduct of Theorem 17.

Corollary 18. Let $\beta \in\{0,1 / 2\}, \Psi$ be a positive function on $(0,2), \mu$ be a positive Borel measure on $\mathbb{D}$ and $\sigma, u \in L_{\mu}^{1}$ non-negative. Then $P_{\Psi, \mu}^{\beta}(\sigma \cdot): L_{\mu}^{p}(\sigma) \rightarrow L_{\mu}^{p}(u)$ is bounded if and 
only if there exist constants $C_{0}=C_{0}(p, \mu, \sigma, u)>0$ and $C_{0}^{\star}=C_{0}^{\star}(p, \mu, \sigma, u)>0$ such that

$$
\left\|\sum_{\substack{I \in \mathcal{D}^{\beta} \\ I \subset I_{0}}}\left\langle\sigma 1_{S\left(I_{0}\right)}, \frac{\Psi(|I|) 1_{S(I)}}{|I|}\right\rangle_{L_{\mu}^{2}} 1_{S(I)}\right\|_{L_{\mu}^{p}(u)}^{p} \leq C_{0} \mu \sigma\left(S\left(I_{0}\right)\right),
$$

and

$$
\left\|\sum_{\substack{I \in \mathcal{D}^{\beta} \\ I \subset I_{0}}}\left\langle u 1_{S\left(I_{0}\right)}, \frac{\Psi(|I|) 1_{S(I)}}{|I|}\right\rangle_{L_{\mu}^{2}} 1_{S(I)}\right\|_{L_{\mu}^{p^{\prime}(\sigma)}}^{p^{\prime}} \leq C_{0}^{\star} \mu u\left(S\left(I_{0}\right)\right),
$$

for all $I_{0} \in \mathcal{D}^{\beta}$. Moreover, there exists a constant $C_{1}>0$ independent of the weights, such that

$$
\left\|P_{\Psi, \mu}^{\beta}(\sigma \cdot)\right\|_{L_{\mu}^{p}(\sigma) \rightarrow L_{\mu}^{p}(u)} \leq C_{1}\left(C_{0}+C_{0}^{\star}\right) .
$$

Proof of Theorem [16. By the equivalence of (A) and (C), $P_{\Psi, \mu}^{+}: L_{\mu}^{p}(v) \rightarrow L_{\mu}^{p}(u)$ is bounded if and only if $\mathcal{M}_{u^{1 / p}} P_{\Psi, \mu}^{+} \mathcal{M}_{\sigma^{1 / p^{\prime}}}: L_{\mu}^{p} \rightarrow L_{\mu}^{p}$ is bounded. By the hypothesis (iv), the adjoint of $\mathcal{M}_{u^{1 / p}} P_{\Psi, \mu}^{+} \mathcal{M}_{\sigma^{1 / p^{\prime}}}$ with respect to the $L_{\mu}^{2}$-pairing is $\mathcal{M}_{\sigma^{1 / p^{\prime}}} P_{\Psi, \mu}^{+} \mathcal{M}_{u^{1 / p}}$. Consequently, the necessity of the conditions (5.1) and (5.2) is obvious. Conversely, by the first inequality in (3.6), the testing conditions (5.1) and (5.2) imply the corresponding testing conditions for each $P_{\Psi, \mu}^{\beta}, \beta \in\{0,1 / 2\}$, that is, conditions (15.15) and (5.16) , and therefore the boundedness of each operator $P_{\Psi, \mu}^{\beta}(\sigma \cdot): L_{\mu}^{p}(\sigma) \rightarrow L_{\mu}^{p}(u), \beta \in\{0,1 / 2\}$, by Corollary 18. The second inequality in (3.6) now implies the boundedness of $P_{\Psi, \mu}^{+}: L_{\mu}^{p}(\sigma) \rightarrow L_{\mu}^{p}(u)$ with the required norm bounds. Finally, by using the equivalence of (A) and (B) and (3.10), we deduce that $P_{\Psi, \mu}^{+}: L_{\mu}^{p}(v) \rightarrow L_{\mu}^{p}(u)$ is bounded with the claimed norm bound.

\section{REFERENCES}

[1] H. Arrousi, Function and operator theory on Large Bergman spaces, doctoral thesis, University of Barcelona, (2016).

[2] A. Aleman, S. Pott and M.C. Reguera, Sarason conjecture on the Bergman space, preprint, available on http://arxiv.org/abs/1304.1750.

[3] D. Bekollé and A. Bonami, Inégalités á poids pour le noyau de Bergman, (French) C. R. Acad. Sci. Paris Sér. A-B 286 (1978), no. 18, 775-778.

[4] D. Bekollé, Inégalités á poids pour le projecteur de Bergman dans la boule unité de $C^{n}$, Studia Math. 71 (1981/82), 305-323.

[5] D. Bekollé, Projections sur des espaces de fonctions holomorphes dans des domaines plans, Canad. J. Math. 38 (1986), 127-157.

[6] O. Constantin and J. A. Peláez, Boundedness of the Bergman projection on $L^{p}$-spaces with exponential weights, Bull. Sci. Math. 139 (2015), 245-268.

[7] Y. Deang, L. Huang, T. Zhao and D. Zeng, Bergman projection and Bergman spaces, J. Operator Theory 46 (2001), no. 1, 3-24.

[8] J. B. Garnett and P. Jones, BMO from dyadic BMO, Pacific J. Math. 99 (1982), no. 2, 351—371.

[9] T. Mei, BMO is the intersection of two translates of dyadic BMO, C. R. Math. Acad. Sci. Paris 336 (2003), no. $12,1003-1006$.

[10] F. Nazarov, S. Treil and A. Volberg, The Bellman functions and two-weight inequalities for Haar multipliers, J. Amer. Math. Soc. 12 (1999), no. 4, 909-928.

[11] J. A. Peláez and J. Rättyä, Weighted Bergman spaces induced by rapidly increasing weights, Mem. Amer. Math. Soc. 227 (2014), no. 1066.

[12] J. A. Peláez and J. Rättyä, Two weight inequality for Bergman projection, J. Math. Pures. Appl. 105 (2016), no. 1, 102-130.

[13] J. A. Peláez and J. Rättyä, Two weight inequality for Bergman projection II, preprint.

[14] J. A. Peláez, Small weighted Bergman spaces, Proceedings of the summer school in complex and harmonic analysis, and related topics, (2016). 
[15] S. Pott and M. C. Reguera, Sharp Bekollé esrtimate for the Bergman projection, J. Funct. Anal. 265 (2013), 3233-3244.

[16] E. T. Sawyer, A characterization of a two-weight norm inequality for maximal operators, Studia Math. 75 (1982), no. 1, 1-11.

[17] E. T. Sawyer, A characterization of two weight norm inequalities for fractional and Poisson integrals, Trans. Amer. Math. Soc. 308 (1998), no. 2, 533-545.

[18] R. L. Schilling, R. Song and Z. Vondraćek, Bernstein functions theory and applications, 2nd Edition, De Gruyter, Studies in Mathematics 37.

[19] S. Shimorin, An integral formula for weighted Bergman Reproducing kernels, Comp. Var., 47 (2002), no. 11 1015-1028.

[20] A. Shields and D. Williams, Bounded projections and the growth of harmonic conjugates in the unit disc, Michigan Math. J. 29 (1982), no. 1, 3-25.

[21] S. Treil, A Remark on Two Weight Estimates for Positive Dyadic Operators, (2012), http://www.arxiv.org/abs/1201.1455.

[22] D. V. Widder, Necessary and sufficient conditions for the representation of a function as a Laplace integral. Trans. Amer. Math. Soc. 33 (1931), 851-892.

[23] Y. E. Zeytuncu, $L^{p}$-regularity of weighted Bergman projections, Trans. Amer. Math. Soc. 365 (2013), 2959-2976.

[24] K. Zhu, Operator Theory in Function Spaces, Second Edition, Math. Surveys and Monographs, Vol. 138, American Mathematical Society: Providence, Rhode Island, 2007.

José Ángel Peláez, Departamento de Análisis Matemático, Universidad de Málaga, Campus de Teatinos, 29071 Málaga, Spain

E-mail address: japelaez@uma.es

Jouni Rättyä, University of Eastern Finland, P.O.Box 111, 80101 Joensuu, Finland

E-mail address: jouni.rattya@uef.fi

Brett D. Wick, Department of Mathematics, Washington University - St. Louis, One BrookIngs Drive, St. Louis, MO USA 63130

E-mail address: wick@math.wustl.edu 